\title{
Recent advances in molecular machines based on toehold-mediated strand displacement reac- tion
}

\author{
Yijun Guo ${ }^{1, \dagger}$, Bing $\mathrm{Wei}^{1,2, \dagger}$, Shiyan $\mathrm{Xiao}^{1}$, Dongbao Yao ${ }^{1}$, Hui $\mathrm{Li}^{1}$, Huaguo $\mathrm{Xu}^{3}$, Tingjie Song ${ }^{1}$, Xiang $\mathrm{Li}^{1}$ and \\ Haojun Liang ${ }^{1,2, *}$ \\ ${ }^{1}$ CAS Key Laboratory of Soft Matter Chemistry, iChEM (Collaborative Innovation Center of Chemistry for Energy Materials), \\ Department of Polymer Science and Engineering, University of Science and Technology of China, Hefei 230026, China \\ 2 Hefei National Laboratory for Physical Sciences at the Microscale, University of Science and Technology of China, Hefei \\ 230026, China \\ ${ }^{3}$ College of Materials and Textile Engineering institute, Jiaxing University, Jiaxing 314000, China \\ * Correspondence: hjliang@ustc.edu.cn
}

Received September 30, 2016; Revised January 11, 2017; Accepted January 18, 2017

\begin{abstract}
Background: The DNA strand displacement reaction, which uses flexible and programmable DNA molecules as reaction components, is the basis of dynamic DNA nanotechnology, and has been widely used in the design of complex autonomous behaviors.

Results: In this review, we first briefly introduce the concept of toehold-mediated strand displacement reaction and its kinetics regulation in pure solution. Thereafter, we review the recent progresses in DNA complex circuit, the assembly of AuNPs driven by DNA molecular machines, and the detection of single nucleotide polymorphism (SNP) using DNA toehold exchange probes in pure solution and in interface state. Lastly, the applications of toehold-mediated strand displacement in the genetic regulation and silencing through combining gene circuit with RNA interference systems are reviewed.

Conclusions: The toehold-mediated strand displacement reaction makes DNA an excellent material for the fabrication of molecular machines and complex circuit, and may potentially be used in the disease diagnosis and the regulation of gene silencing in the near future.
\end{abstract}

Keywords: toehold-mediated strand displacement; DNA molecular machines; SNP; gene expression regulation

\section{INTRODUCTION}

Because of its particular composition diversity and predictable complementary base pairing principle for molecular recognition, DNA attracts wide attention as a powerful and versatile material and has been effectively applied in genetic detection and treatment, achieving distinct nanostructures in terms of self-assembly. Moreover, the specific interactions between DNA and metal ions, small organic molecules, polypeptide chains, even

\footnotetext{
These authors contributed equally to this work.

This article is dedicated to the Special Collection of Synthetic Biology, Aiming for Quantitative Control of Cellular Systems (Eds. Cheemeng Tan and Haiyan Liu).
}

proteins, enable the possibility to establish sophisticated nanostructures and nanodevices using DNA.

After the first introduction of the concept of toeholdmediated strand displacement by Yurke et al. [1] in the early 21 st century, booming developments have been achieved in the field of dynamic DNA nanotechnology. For toehold-mediated strand displacement, the single stranded invading oligomer strand, first binds to the overhanging complementary domain (referred as the toehold domain) of the double stranded complex constructed using the substrate and protector strands, then initiates the strand displacement through branch migration, and finally releases the prehybridized protector strand. The first DNA molecular machine was reported by Yurke and co-workers who constructed a molecular 
tweezer made from three strands of DNA [1]. This DNA molecular tweezer can be opened and closed repeatedly by cycling addition of auxiliary strands of "fuel" DNA, and each cycle produces a duplex DNA waste product. Until recent, the toehold-mediated strand displacement reaction has been vastly used in designing molecular devices [2], autonomous walkers [3], logic robot [4], molecular automata [5], and structures [6,7] for molecular diagnostics [8], and mimics of complex biological processes [9]. Moreover, DNA dynamic nanotechnology provides a practical method for synthetic biology [10] through the programmable structural transformation of biologically related nucleic acids. DNA as genetic material can be transcribed to form messenger RNA (mRNA) for managing gene expression regulated by various biological molecules, especially proteins.

In this paper, we will first discuss the mechanism understanding of and kinetic regulation of toeholdmediated strand-displacement reaction, and then review the progress in the construction of diverse nanostructures or nanodevices based on DNA molecular machines, such as logic circuits [11], innovative DNA nanoparticle (NP) complexes [12], molecule probes [13], and single-base discrimination biosensors $[14,15]$. Lastly, we will discuss the RNA-based riboregulator and conditional RNA interference applied to control gene expression.

\section{TOEHOLD-MEDIATED DNA STRAND DISPLACEMENT REACTION-BASED DYNAMIC DNA SYSTEMS}

In toehold-mediated strand displacement reactions, a single-stranded domain first binds to the dangling toehold domain of a pre-hybridized double-strand (substrate), triggers the branch migration, and results in the dissociation of the third strand previously bound to the substrate. The toehold domain plays an important role in facilitating strand displacement, and the length of toehold is often used to control its reaction kinetics [16]. In general, increasing the toehold length will speed up the rate of strand displacement because of the stronger binding energy, that is, a long toehold will thermodynamically enhance the energy difference between products and reactants. To weaken the strong coupling between the kinetics and the thermodynamics of strand displacement, a reaction called "toehold exchange" was first proposed by Zhang and Winfree to improve the control of strand displacement kinetics (Figure 1) [1]. More importantly, the activated but formerly sequestered domain in the substrate resulting from the toehold exchange reaction, in combination with the reaction's reversibility, enables the possibility to construct large reaction networks [16].

\section{Mechanism understanding of DNA strand displacement}

Based on the calculations of Zhang and winfree, DNA strand displacement can be modeled as biomolecular reactions and modulated kinetically (Figure 2) [17,18], which facilitate the prediction of kinetics of scaled-up and multilayer circuits. In addition, mismatched base pairs [19] are also introduced for kinetic modeling and control of DNA strand displacement.

The control of DNA devices and circuits abased on conventional toehold-mediated strand displacement reactions is limited within the tube spatially, because the systems begin to operate after the addition of trigger strands. For further regulation with external control, toeholds are often deliberately "hidden" via various "coverings" (Figure 3), such as hybridization [20], bulge-loop structure [21], hairpin [22], nucleobase-caging groups [23,24], or parallel Hoogsteen motif [25], which are responsive to environmental stimuli, including light and $\mathrm{pH}$. Similar to the conception of "hidden", the idea of split was also introduced by distributing toeholds and BM domains on different strands to break the "hard-wired" combination between them and make the circuit design flexible. Tied up via hybridization of additional domains, "associative toeholds" (proposed by Chen, Figure 4A, 4B [26]) form in a three-way junction manner, and the formation of toeholds driven by junction structures efficiently activate DNA circuits, such as self-replicator [26] and logic circuits [27,28]. If toeholds and BM domains are distributed on the same strands, another type of split termed "remote toehold" (Figure 4C, 4D) forms [29], which allows additional control over strand displacement kinetics by tuning spacer regions.

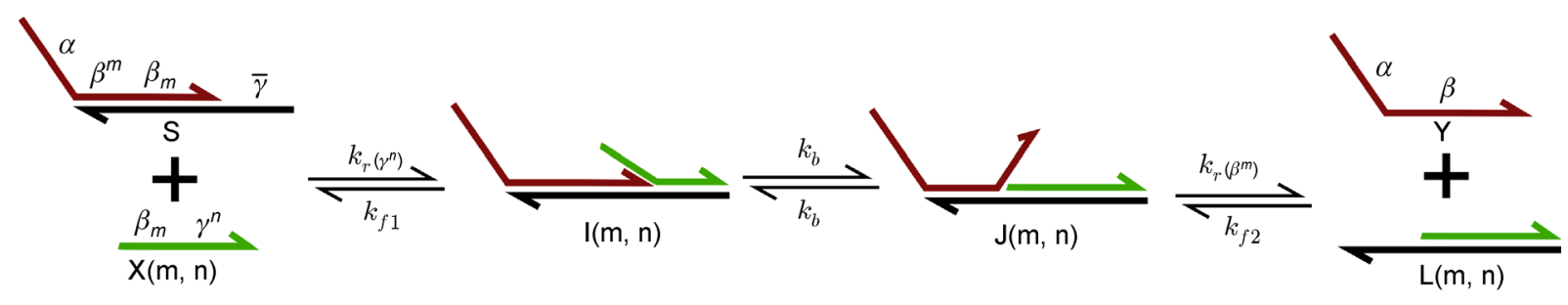

Figure 1. Mechanism of toehold exchange reaction. 

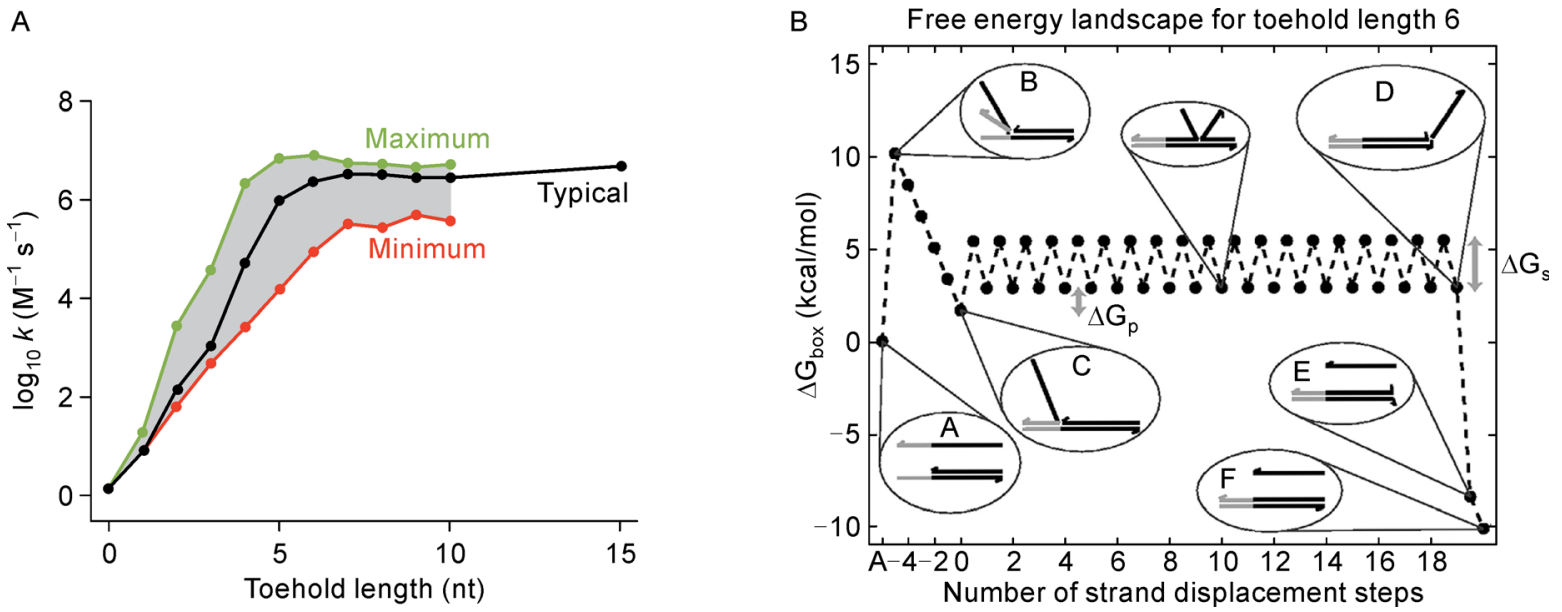

Figure 2. Toehold control and Intuitive Energy Landscape (IEL) model of DNA strand displacement. (A) Experiments show that kinetics of strand displacement depend on sequence and length of toeholds. The "Maximum" (green) shows the kinetics of strand displacement mediated by strong toehold (only G/C nucleotides), whereas the "Minimum" (red) shows that of weak toehold (only A/T nucleotides), and the "Typical" (black) shows that of toehold with equal numbers of all four nucleotides; reprinted with permission from Ref. [17], Copyright 2009 American Chemical Society. (B) The IEL models strand displacement by dividing the free energy into several states $(\mathrm{A}-\mathrm{F})$ with the parameters of sawtooth amplitude $(\Delta \mathrm{Gs})$ and plateau height $(\Delta \mathrm{Gp})$; reprinted with permission from Ref. [18], Copyright 2013 Oxford University Press.

A

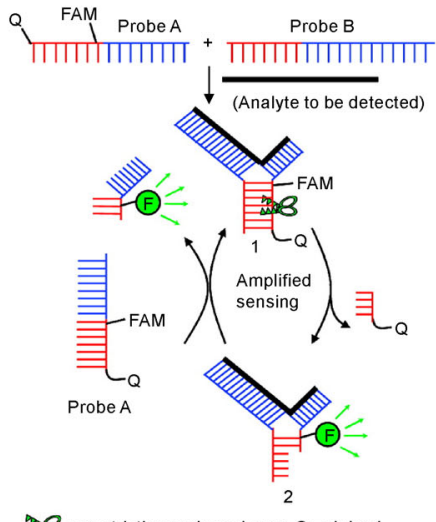

B

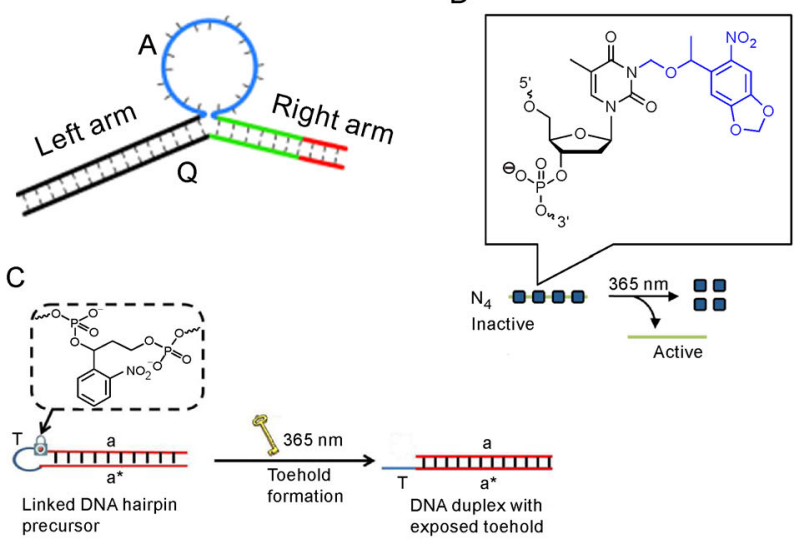

$\mathrm{E}$

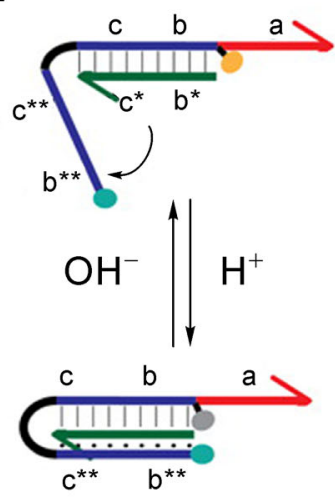

Figure 3. Various "coverings" for toehold hiding. Toeholds are hidden by (A) hybridization; reprinted with permission from Ref. [20], Copyright 2008 American Chemical Society. (B) Bulge-loop structure; reprinted with permission from Ref. [21], Copyright 2011 WILEY-VCH Verlag GmbH \& Co. KGaA, Weinheim. (C) Hairpin; reprinted with permission from Ref. [22], Copyright 2013 American Chemical Society. (D) Nucleobase-caging groups; reprinted with permission from Ref. [24], Copyright 2013 American Chemical Society. (E) Parallel Hoogsteen motif; reprinted with permission from Ref. [25], Copyright 2014 American Chemical Society.

\section{Kinetics control of the toehold-mediated strand displacement reaction}

According to the suggestions proposed by Zhang and Winfree [16], the toehold exchange reaction (Figure 1) can be modeled as a simple biomolecular reaction. It can be characterized using the three-step model to derive its biomolecular reaction constant as follows:

$$
k_{\left(\beta^{m}, \beta_{m}, \gamma^{n}\right)}=\frac{k_{r\left(\beta^{m}\right)} k_{f} k_{b}}{k_{r\left(\gamma^{n}\right)} k_{r\left(\beta^{m}\right)}+k_{r\left(\gamma^{n}\right)} k_{b}+k_{r\left(\beta^{m}\right)} k_{b}}
$$

where $k_{f}$ is the hybridization rate, and $k_{r\left(\gamma^{n}\right)}$ and $k_{r\left(\beta^{m}\right)}$ are the first-order of toeholds $\beta^{m}$ and $\gamma^{n}$ dissociating from their complements, respectively. Moreover, the predicted biomolecular rate constant depends on the toehold binding energies, which can be calculated based on 
A

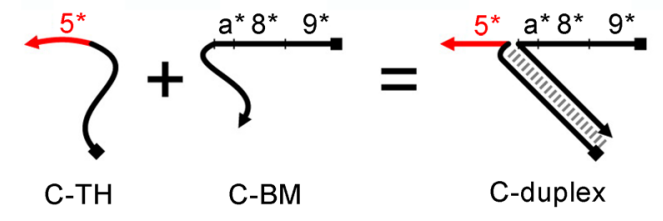

C

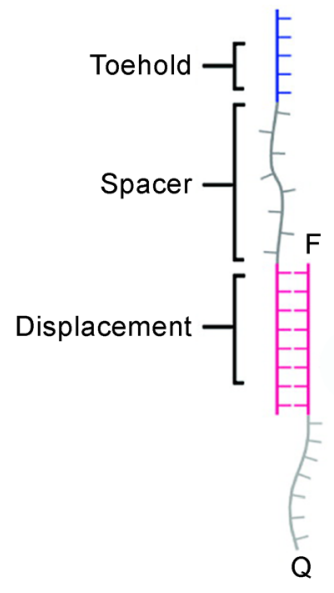

D
B

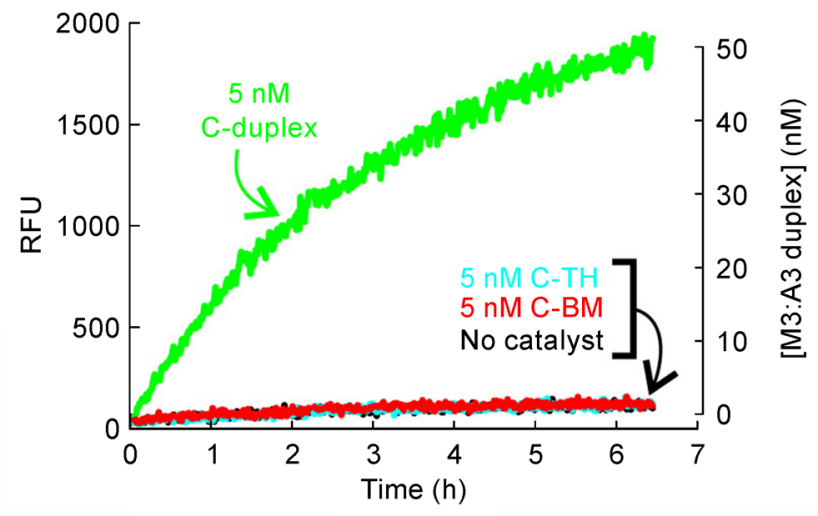

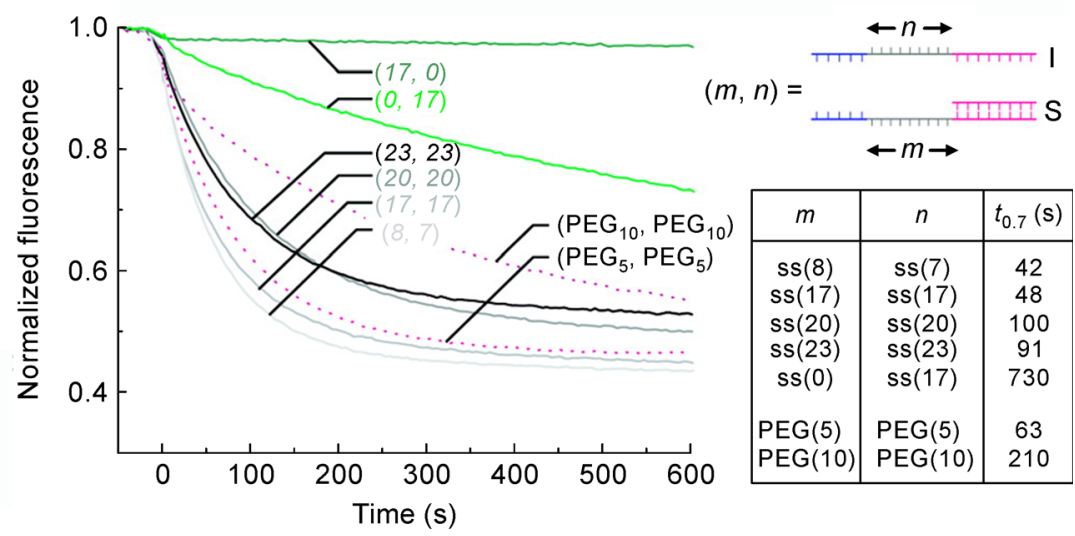

Figure 4. Toehold split schemes. (A) The toehold domain and the branch migration (BM) domain were distributed on different strands (C-TH and C-BM), and they formed the "associative toehold" (C-duplex) via additional hybridization. (B) Experiment results of the strand displacement mediated by "associative toehold". (A) and (B), reprinted with permission from Ref. [26], Copyright 2012 American Chemical Society. (C) The toehold domain and the BM domain were distributed on the same strand and interspaced by a spacer domain, leading to the formation of "remote toehold". (D) Experiment results of the strand displacement mediated by "remote toehold". (C) and (D), reprinted with permission from Ref. [29], Copyright 2011 American Chemical Society.

initiation energy and base stacking energy parameters [30-34]. The reaction rate of strand displacement varies exponentially with the binding strength of toehold domain; theoretical calculations and experiments indicate that the biomolecular rate of the toehold exchange reaction can be tuned by $10^{6}$-fold by modulating the length and sequence of toehold domain $[1,17,35]$.

Given the inaccuracy and incompleteness in nucleic acid thermodynamic parameters and detailed biophysical understanding of strand displacement kinetics [18], fine control of the kinetics of DNA strand displacement remains difficult, thereby limiting its ability to program toehold exchange reactions into complex DNA circuits. To address this problem, Genot and coworkers introduced a spacer between the toehold and displacement domains, which is referred to as remote toehold, to provide additional control to the strand displacement rates over at least three orders of magnitude by adjusting the length of spacer [29]. The kinetics of strand displacement can also be controlled by introducing a mismatch at the incumbent domain, and altering the position of defect can tune the reaction kinetics across three orders of magnitude [19]. Yang and his coworkers proposed an allosteric DNA toehold (A-toehold) design that allows the flexible regulation of DNA strand displacement by splitting an input strand into an A-toehold and branch migration domain. This A-toehold design enables the dynamic selective activation of multiple strand displacement reactions [36]. Considering the critical role of solvent in the stability of DNA structural properties [37,38], organic solvents were used by Liu, Xia, and their coworkers to adjust the kinetics of DNA hybridization [39-41]. Their studies indicated that the hybridization kinetics of the DNA beacon progressively rise as the ethanol content in solvent increases, and a 70-fold rate enhancement is achieved for DNA in a solvent with 56\% ethanol [40]. Similar behavior was also observed for other types of organic solvents. In contrast to the methodology of kinetics control by introducing a defect or spacer in the DNA substrate, the concentration percentage of organic 
molecules in solvent can be continuously tuned, therefore we may continuously modulate the hybridization kinetics. Similarly, Zhang and his coworkers recently proposed the "stoichiometric tuning" method to adjust yield and selectivity using the relative concentration of an auxiliary species for hybridization probes, achieving near-continuous tuning of the probe and effective free energy [42].

\section{APPLICATIONS OF DYNAMIC DNA MOLECULAR MACHINES}

\section{Engineering the assembly of gold nanoparticles (AuNPs) driven by DNA molecular machines}

The fabrication of well-defined architecture, such as spatially arranging NPs in a predetermined manner, is a classical bottom-up approach in modulating the performance of devices. Since two pioneering groups realized the assembly of DNA-modified AuNPs by utilizing the base-paring interactions of oligonucleotides in 1996 $[43,44]$, an increasing number of researchers have focused on programming the assembly of AuNPs with DNA strands for potential applications in sensing, diagnosing, and constructing optical devices.

In 2008, Mirkin, Gang and their coworkers reported DNA-programmable NP crystallization for the first time $[45,46]$. Furthermore, basic design rules on DNA sequences and length were established [47] to obtain ordered NP arrays in three dimensions (Figure 5) $[48,49]$. Meanwhile, developing robust DNA framework for adjusting the spatial distribution of NPs has attracted much attention. Yan et al. demonstrated that the two- and three-dimensional arrangement of NPs can be modulated with DNA origami [50], on which the position of NPs can be precisely located. Furthermore, accurate positioning of AuNPs was achieved by integrating different origami structures with distinct shapes, and the plasmonic properties of the final nanostructures were finely modulated [51,52]. Recently, Gang's group proved that prescribed NP architectures can be efficiently constructed with assorted DNA frames [53,54].

Besides the progress of DNA-based assembly of NPs in the field of materials science[55], this assembly strategy also plays pivotal roles in sensing [56-58]. In the traditional assembly strategy, the aggregation of DNAfunctionalized NP is initiated with the addition of a linker strand, which can be treated as a target in the sensing system. The construction of DNA/RNA sensors on the basis of NP assembly has been intensively explored in past decades [59]. To further expand the sensing capacity on small molecules and proteins using the traditional method, functional DNA sequences including DNAzyme and aptamer were integrated for building a responsive system in vitro [60] and in vivo [61,62].

However, traditional DNA sensors have their own limitation in detection because of the continuous consumption of targets. To address this issue, Liang's group proposed a new method for engineering the assembly of DNA-modified NPs [12]. In their methodology, the linker strand was pre-hybridized with a protector
A
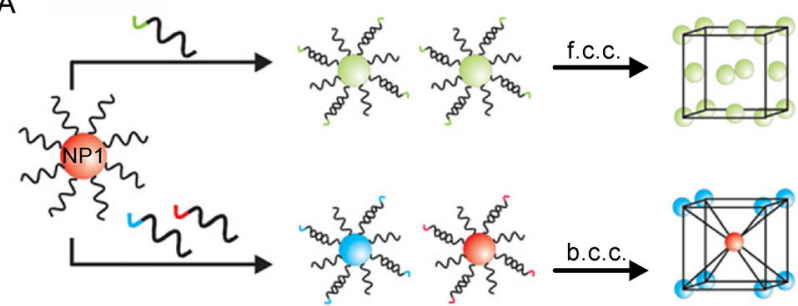

B

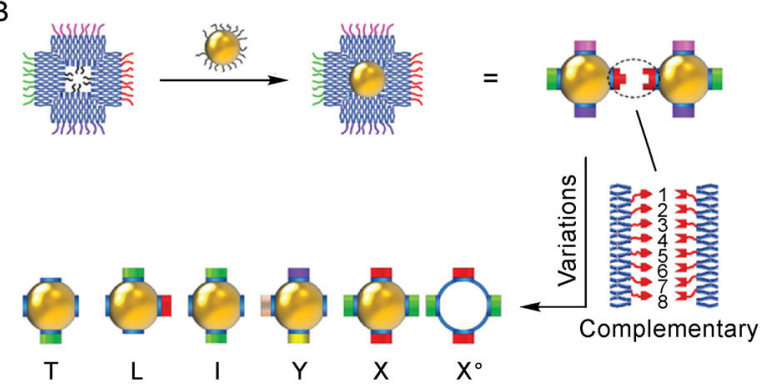

C
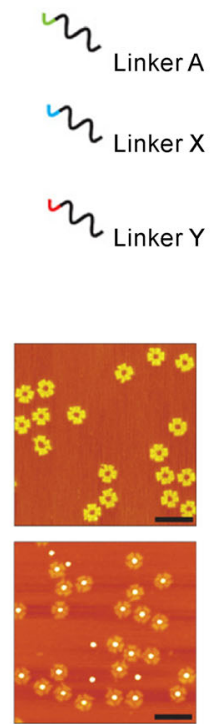
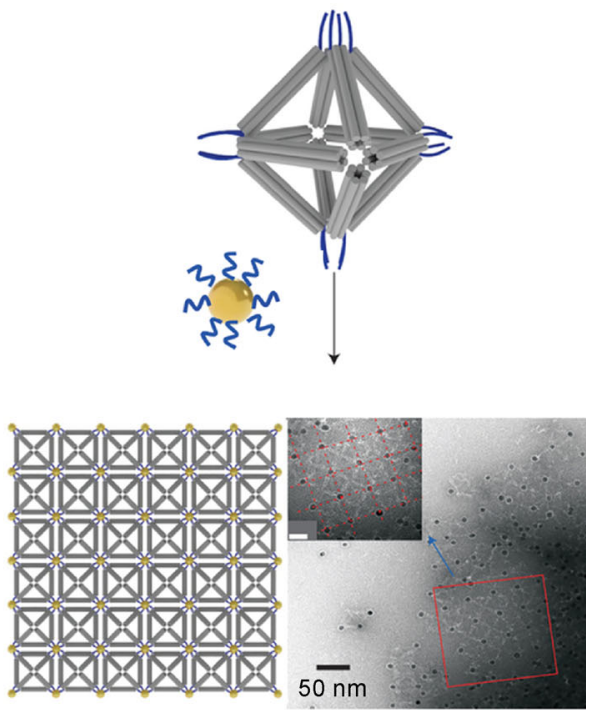

Figure 5. DNA-AuNP assembly. The architect of prescribed nanoparticle arrays through programming the (A) primary; reprinted with permission from Ref. [46], Copyright 2008 Nature Publishing Group. (B, C) three-dimensional structures of DNA. (B), reprinted with permission from Ref. [48], Copyright 2015 Nature Publishing Group. (C), reprinted with permission from Ref. [49], Copyright 2016 Nature Publishing Group. 
strand, so direct conjugation between two kinds of NPs was inhibited. The exogenous target strands were introduced to initiate a series of toehold-mediated strand displacement reactions and activate the function of linker strand. Finally, the assembly of NPs, accompanied with the change in colorimetric signal, was achieved (Figure 6). Based on the above procedures, the limitation in DNA detection can be efficiently decreased compared with the traditional strategy. Moreover, this method can be applied to construct more complexed and robust machines, such as multiple-component logic gates for multiplexed DNA detection.

\section{Discrimination of single nucleotide polymorphism (SNP)}

SNP is one of the most common human heritable variations. Discriminating single-base changes in human DNA is highly desirable, because SNP discrimination is essential to the diagnosis of genetic diseases. To date, diverse tools including polymerase chain reaction (PCR) [63], microarrays [64,65], and modern sequencing-bysynthesis [66] have been developed for detecting such single nucleotide variants (SNVs).

In 2012, Zhang, Chen, and Yin designed a toehold exchange probe based on the theoretical framework of hybridization thermodynamics; the probe can robustly discriminate single-base changes and ensure near-optimal specificity across concentrations, temperatures, and salinities [67]. The toehold exchange probe $(P C)$ consists of a substrate $C$ pre-hybridized by a protector strand $P$ while holding a handling toehold with a seven-base domain. The target strand $X$ can react with the substrate with the help of toehold to release $P$ and produce $X C: X+$ $P C \rightarrow P+X C$. For the toehold exchange probe, no number change of species occurs between the reactants and products, which means that the change in the number of species $(\Delta n)$ equals zero. Therefore, the hybridization yield $\chi$ is not dependent on the initial concentration of the limiting reagent (c). Moreover, $\Delta H^{0}$ and $\Delta S$ of hybridization are assumed temperature invariant under the standard thermodynamic modes of DNA hybridization. The probe is designed with its standard free energy change $\left(\Delta G^{0}\right)$ close to zero for the intended target $(\chi \approx 0.5)$, and hybridization between the spurious target $(S)$ with single base change and the substrate $(P C)$ is less thermodynamically favorable by $\Delta \Delta G^{0}$. The discrimination factor is defined as $Q=\chi_{x} / \chi_{s}$; theoretically $Q_{\max }=\exp \left(\Delta \Delta G^{0} /\right.$ $R T)$. This probe was tested comprehensively and achieved discrimination factors between 3 and $100+$, with a median value of 26 . More importantly, the probes showed good robustness across a wide range of temperature, salinity, and oligonucleotide concentration [67].

The hybridization probe was further improved in 2013 by Chen, Zhang, and Seelig [68]. They presented a new class of conditionally fluorescent molecular probes, which
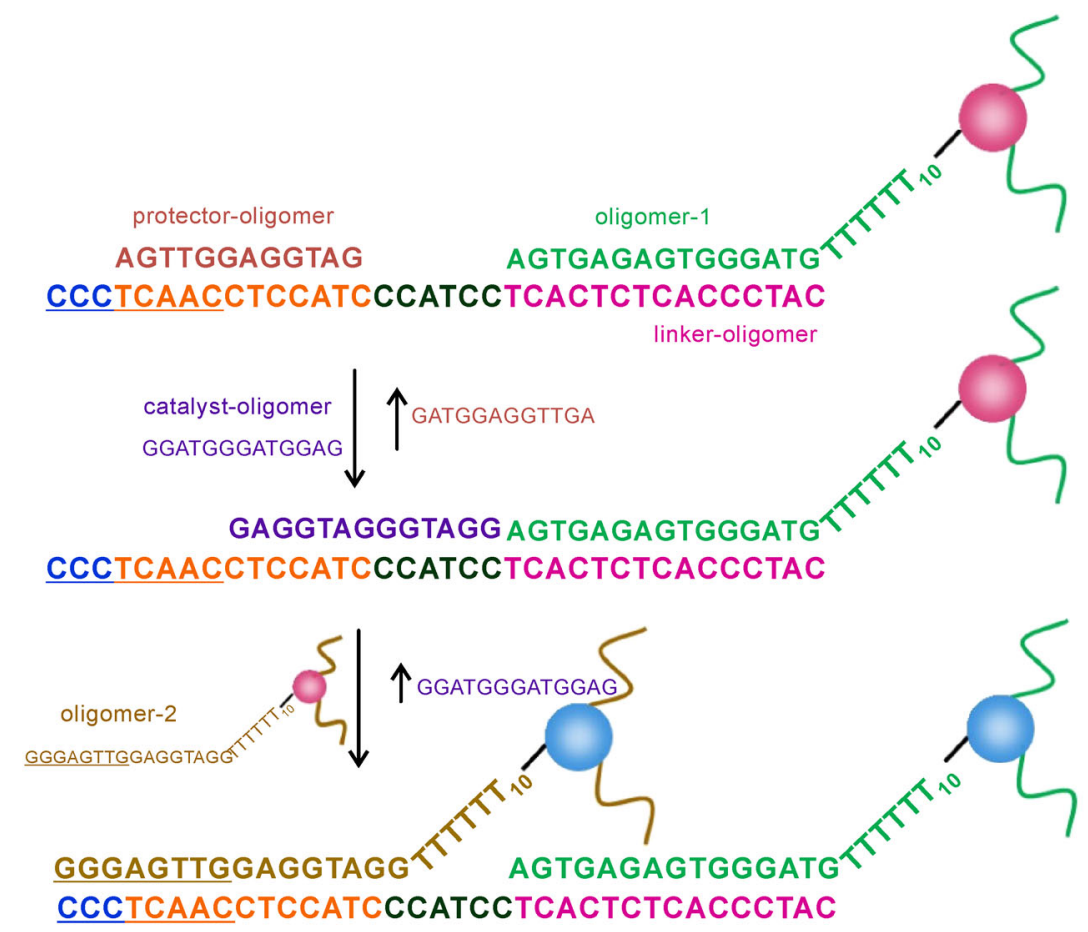

Figure 6. Graphical representation of the dynamic DNA-fueled molecular machine strategy and the mechanism of DNAAuNP assembly. Reprinted with permission from Ref. [12], Copyright 2012 American Chemical Society. 
can effectively discriminate single base changes in double-stranded DNA (dsDNA). This approach is designed based on the mechanism of double-stranded toehold exchange. This novel mechanism can generate two thermodynamically destabilizing mismatch bubbles and effectively discriminate the single-base change in the target. The competitive composition was first introduced by Wang and Zhang in 2015 [69] to improve the accuracy of hybridization-based approaches for analyzing nucleic acids. The competitive composition comprises a targetspecific probe and wild-type (WT) sink molecule, which is specific to the WT strand. Simulation is used to optimize the combination of thermodynamic parameters of the probe and sink, as well as achieve extremely high selectivity in generating normalized fold changes in excess of 200 for all tested 44 SNV targets, thereby suggesting high molecular specificity. Thereafter, Chen and Seelig [70] combined the competition with amplification to identify mutations further away from the toehold domain, which is beyond the capability of pure amplification or competition probes. The newly proposed probe functions in a two-step mechanism as follows:

(i) $I_{T}+P_{\text {amp }} \stackrel{k_{f}}{\rightarrow} I_{T}+F_{T}$, and (ii) $I_{T}+S \stackrel{k_{s}}{\rightarrow} W_{T}$. $I_{T}$ is the intended target, $P_{\mathrm{amp}}$ is the amplification probe, and $S$ is the sink. The discrimination factor can be theoretically derived by using the concentrations of fluorescent signal species in the case of intended and spurious targets $\left(\left[F_{T}\right]\right.$ and $\left.\left[F_{S N V}\right]\right)$. Therefore, the probe system possesses quadratically better end point discrimination than the purely competitive system. The experiments verified the theoretical predictions.

\section{SNP discrimination using Dual-polarization interferometry (DPI)}

DPI is one of the most powerful approaches in the variation measurement of the layer at a liquid-solid interface in terms of mass, thickness, and density [71,72].

Liang's group first utilized DPI to monitor the entire process of a toehold-mediated DNA strand displacement reaction in real time, and calculated its efficiency based on the mass changes on the surface [73]. The surface mass changes were examined instantly upon addition of the displacement DNA with variation in toehold lengths and DNA concentrations. The accurate displacement percentage was obtained by comparing the variations in mass. It was found that the displacement efficiency increased with the rising of DNA concentration.

Moreover, Liang's group developed a single-base change discrimination strategy through the toeholdmediated strand displacement reaction on a chip surface by using DPI detection (Figures 7A and 7B) [13]. They carefully compared the efficiencies in solution and on chip surface under the same temperature and salt concentration. As shown in Figure $7 \mathrm{C}$, the reaction on the surface was enhanced greatly by the correct target but only slightly enhanced by the spurious targets. The reaction with the correct target showed an efficiency of $86.1 \%$ on the chip surface but only $24.9 \%$ in solution. These results proved that the strand displacement efficiency on the chip surface was much higher than that in solution under the same condition. Based on this enhanced toehold exchange on the chip surface, a single base difference along the DNA chain was unambiguously discriminated.

\section{SNP discriminated using quartz crystal microbalance (QCM)}

QCM is a powerful tool that realizes real-time, label/labelfree detection of SNPs on a sensing chip including the toehold-mediated strand displacement reaction. For example, Liu et al. proposed a self-assembly of onedimensional DNA nanostructure strategy on the QCM platform based on the strand displacement reaction (Figure 8A) [74]. The target p53 disturbed the capture probe hairpin and unfolded the closed structure. Subsequently, two additional hairpins ( $\mathrm{H} 1$ and $\mathrm{H} 2)$, which were designed to facilitate cross-hybridization to the toehold domain, were introduced to trigger the hybridization chain reaction. According to this design rationale, $\mathrm{H} 1$ and $\mathrm{H} 2$ can be polymerized into a one one-dimensional DNA nanostructure on the chip surface, and this DNA nanostructure functioned as an amplifier for QCM frequency shift. Recently, a new amplified QCM platform using an oligonucleotide target-triggered layer-by-layer assembled DNA-streptavidin dendrimer nanostructure as an efficient amplifier has been developed [75]. The construction and rationale are based on the fact that one SA molecule has four binding sites for coupling with the biotin-modified single-strand DNA (Figure 8B). The target DNA opened the hairpin after immobilization of the capture probe on the chip surface. Sequentially, two building blocks (DNA1-SA and DNA2-SA) were injected to construct a DNA-SA dendrimer nanostructure. This strategy is very simple and specific, with no need for a complex separation process or enzymatic reaction. Additionally, the QCM chip platform can also be applied in DNA logic operations [76] and highly specific detection of single-base DNA mutation [77].

Compared with classic sandwich-type hybridization, the advantage of toehold-mediated DNA assembly is remarkable. As shown in Figure 8, Liang's group developed a method to achieve discrimination of a perfect target from mutant sequences with single-base mismatch, insertion, and deletion on the QCM platform (Figure 9) [78]. This method investigates the optimal lengths of toehold domains $\left(\overline{\gamma^{n}}\right.$ and $\left.\overline{\beta^{m}}\right)$. Finally, they found that $n / m$ $=6 / 8$ is the best choice for this method. In this strategy, 


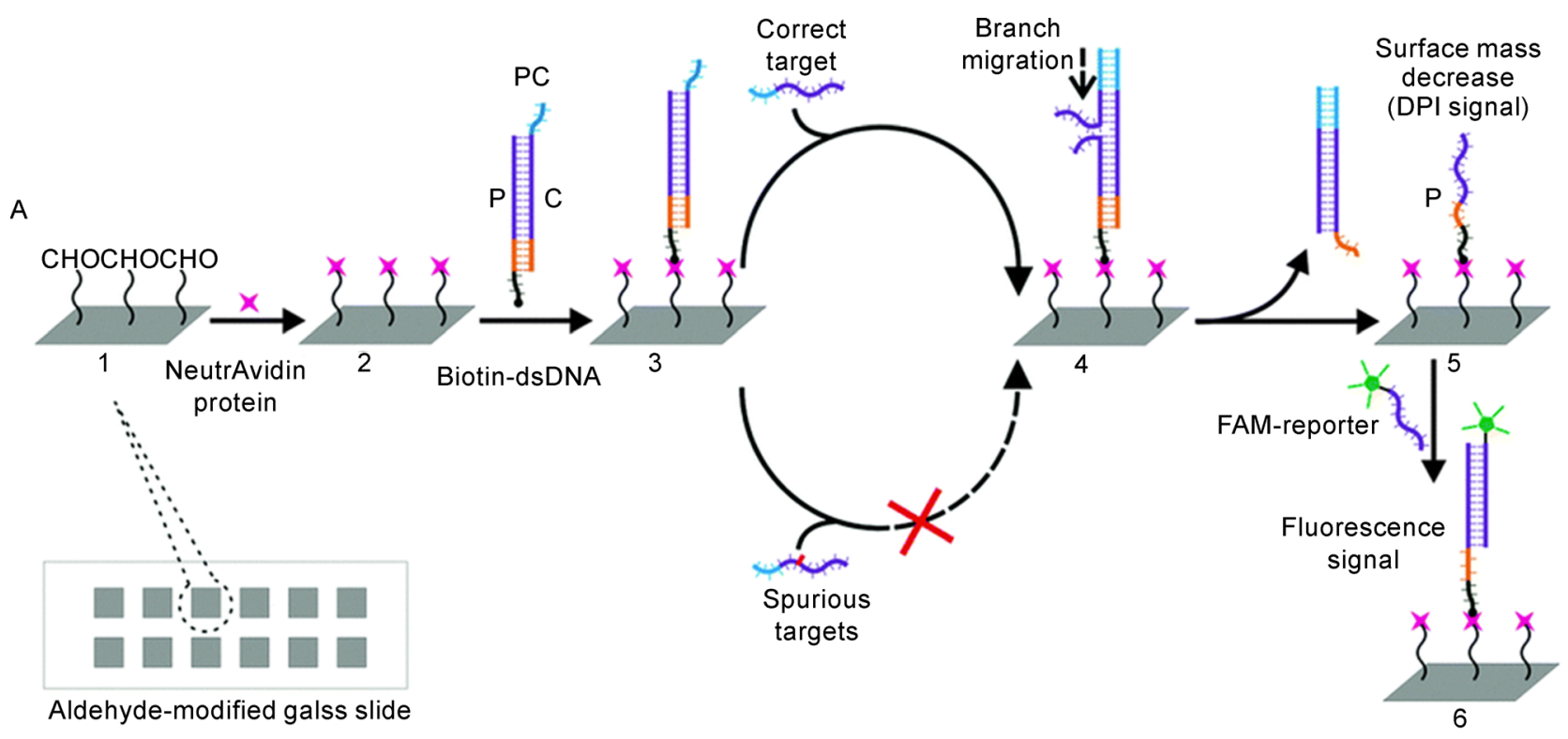

B

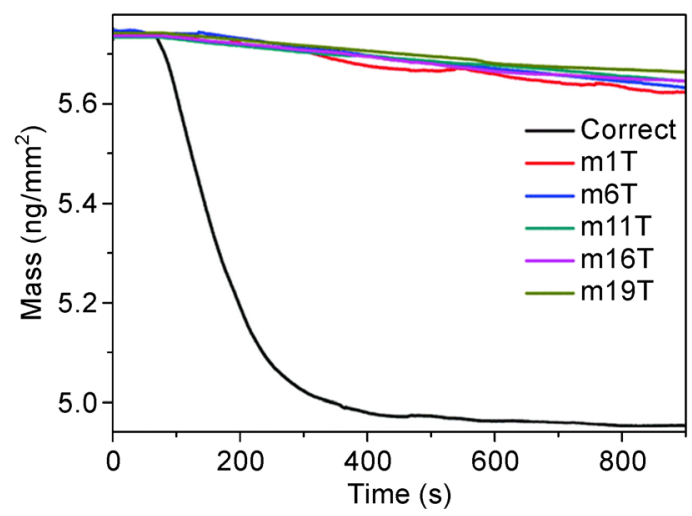

C

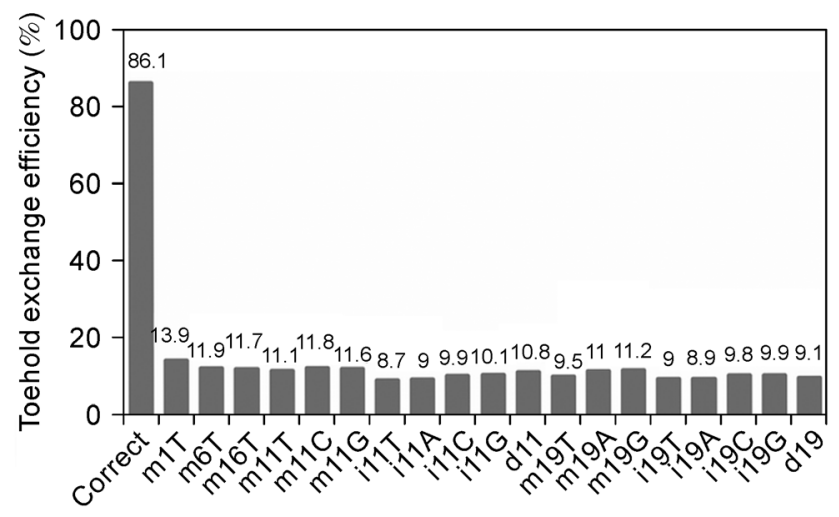

Figure 7. Toehold-mediated strand displacement reaction on the chip surface to discriminate SNP. (A) Schematic representation of the DNA toehold exchange process on chip surface to detect single-base changes. (B) Real-time DPI measurements of surface mass decrease on a sensor chip surface modified with a dsDNA probe (PC) with the addition of correct target ssDNA (Correct). Different spurious targets have a single-base mismatch at different positions (m1T to m19T). (C) Calculated toehold exchange efficiencies on chip surface with the addition of correct target ssDNA and different spurious targets; reprinted with permission from Ref. [13], Copyright 2014 Royal Society of Chemistry.

DNA-AuNP can release the target sequence back into the solution, so it can realize circular initiation of the strand displacement reaction by displacing the target sequence from the linker oligomer. This design is helpful for the sensitivity of this method. Therefore, the DNA-AuNP probe-fueled strand displacement reaction based on QCM can achieve distinct discrimination of a single-base mismatch, which can be used for gene detection.

\section{DNA nanomachine-controlled AuNP assembly- based SNP discrimination}

Although various SNP genotyping methods have been developed $[79,80]$, these methods suffer from some drawbacks, such as complex handling procedures, easy contamination, high cost, and lack of portability. Therefore, developing robust hybridization probes with high specificity is strongly important for nucleic acid biotechnology. Given the distinct optical and chemical properties of AuNP, AuNP-based SNP detection is of particular interest among numerous strategies [81,82].

After Mirkin et al. established the innovative work of DNA-AuNP assembly strategy [43], they first used the AuNP-based colorimetric assay for SNP discrimination [81]. This method can discriminate the correct target with a variety of mutated targets, However, this "direct-linkage strategy" cannot be implemented under isothermal conditions, which may limit its application. In particular, 
A

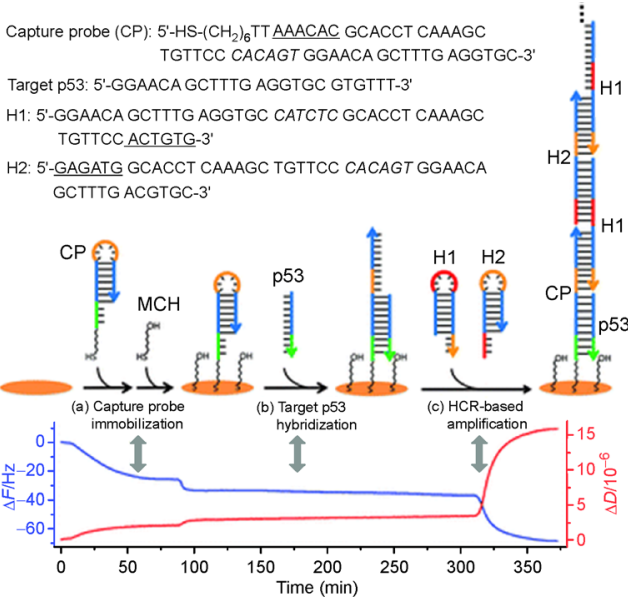

B

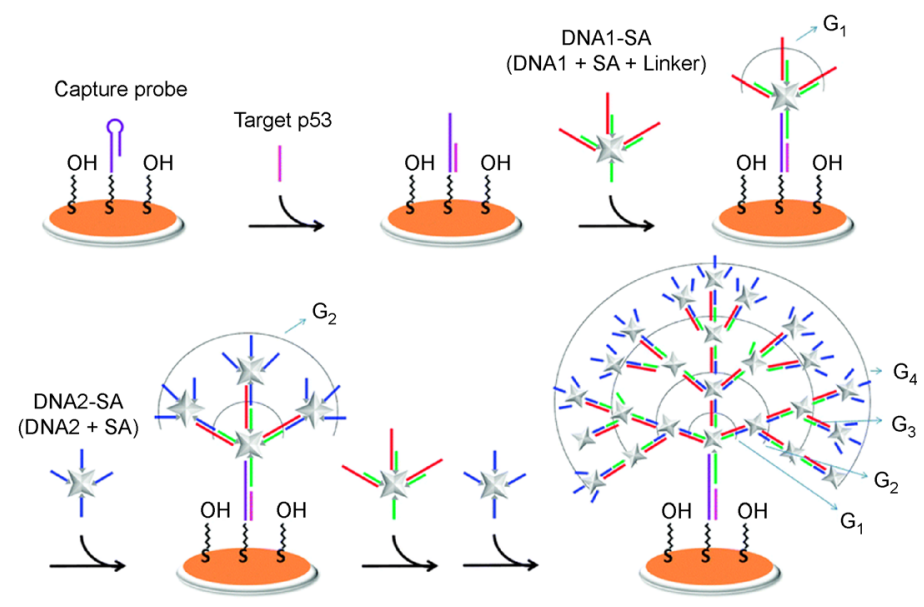

Figure 8. QCM sensing platform application. (A) Schematic representation of the designed QCM-D biosensing platform for realtime detection of target p53 using a self-assembled DNA nanostructure as an efficient signal amplifier. QCM-D response curves show the corresponding frequency and dissipation shifts of $20 \mathrm{nM}$ of target p53 for the above three steps; reprinted with permission from Ref. [74], Copyright 2012 Royal Society of Chemistry. (B) Schematic representation of the construction and rationale of DNA-SA dendrimer-amplified QCM sensing platform; reprinted with permission from Ref. [75], Copyright 2015 Royal Society of Chemistry.

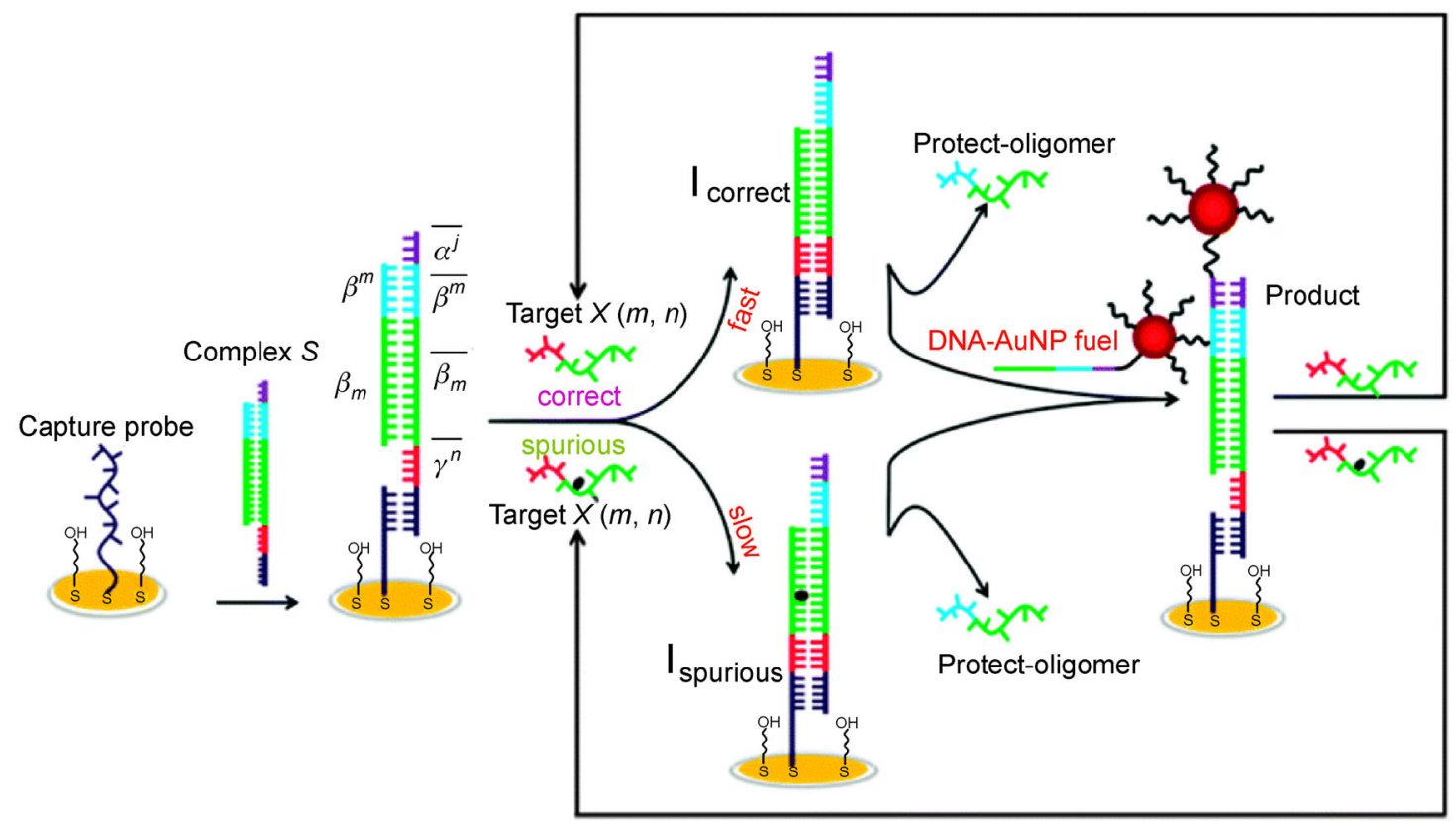

Figure 9. Schematic representation of the design rationale of discrimination of a single-base change on platform of QCM. Reprinted with permission from Ref. [78], Copyright 2015 Royal Society of Chemistry.

the toehold-mediated strand displacement reaction was ingeniously introduced to engineer the catalytic assembly of DNA-AuNPs $[12,14,15,83,84]$. In these systems, DNA-AuNP assembly can be precisely regulated by changing toehold length, sequence composite, and concentration of substrate and target. In addition, the target strands in these systems can be recycled after the reaction, which is more effective compared with the traditional "direct-linkage strategy" $[43,81,82]$.

Based on this new isothermal toehold-mediated strand displacement reaction-based catalytic DNA-AuNP strategy, Liang's group reported an efficient method for the discrimination of single-base changes at room temperature [15]. The graphical representation of the strategy for 
discriminating SNP is displayed in Figure 10A. In this system, two types of DNA-AuNPs are initially in a metastable state in which it is thermodynamically favorable for them to react. Only after the addition of a target strand (called catalyst), the system can be triggered with a series of strand displacement reactions, and the catalyst strand can be recycled in this system. Finally, two kinds of DNA-AuNPs will be assembled into visible aggregates. If the mutated DNA target is added, the reactivity of this system will reduce. Under such conditions, they developed a five-step model based on the toehold exchange reaction of Zhang and Winfree to describe the discrimination for the systems with the addition of intended and spurious targets. For the probe, the discrimination factor can be expressed as $D=10 /\left(1+1 / 6 \times 10^{6-n}+10^{m-n}\right)$, where $n$ and $m$ are the lengths of the invading and incumbent toeholds in the first reaction step, respectively. The performance of the probe in discriminating intended and spurious targets can be optimized by modulating the toehold lengths. Guided by optimization using theory, they improved sensitivity by 10 -fold to 100 -fold over traditional AuNP probes. Therefore, with the precise design of the toehold length in the linker strand, DNA targets with a single-base change can be distinguishable from the correct strand within $4 \mathrm{~h}$ for both SNPs and indels.

Although the established strategy in Figure 10A is effective in the discrimination of single-base changes, the assembly of DNA-AuNPs can only be triggered by specifically designed targets. The significant sequence constraints imposed on the function of DNA-AuNPs constitute a substantial challenge. Considering that the properties of DNA-AuNPs and hybridization on the surface of AuNPs are greatly affected by DNA surface density, NP size and shape, and salt concentration, repeated DNA-AuNP synthesis will introduce uncertain factors that may affect assay analyses.

Therefore, an integrated toehold-mediated strand displacement reaction-based DNA circuitry with selfassembly of DNA-AuNPs was developed [14]. This improved system is composed of two subsystems: the upstream toehold-mediated strand displacement reactionbased catalytic circuit and the downstream DNA-AuNPs (Figure 10B). These two subsystems can be optimized or regulated separately and integrated through a linker oligonucleotide, which is released through the upstream circuit built based on the toehold-mediated strand displacement reaction. In particular, the domains of the strands modified on AuNPs in this system do not participate in reactions occurring in reaction systems. This scenario enables the synthesized DNA-AuNP conjugates to detect arbitrary target DNAs. With this feature, the same DNA-AuNPs are allowed to combine with different toehold-mediated strand displacement reaction-based DNA circuits without re-preparing new DNA-AuNPs, which save laborious work and cost.

With the help of theoretical calculations, they optimized the lengths of toehold to endow the integrated system with the ability to discriminate single-base changes on a sequence of target strand. The toehold strategy for catassembler and protector was $n / m=5 / 5$. For all kinds of mutations (mismatch, insertion, and deletion)
A

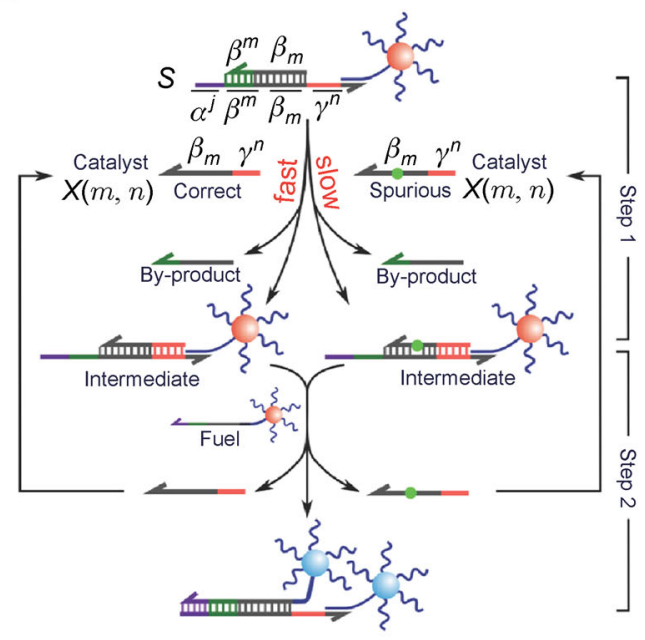

B

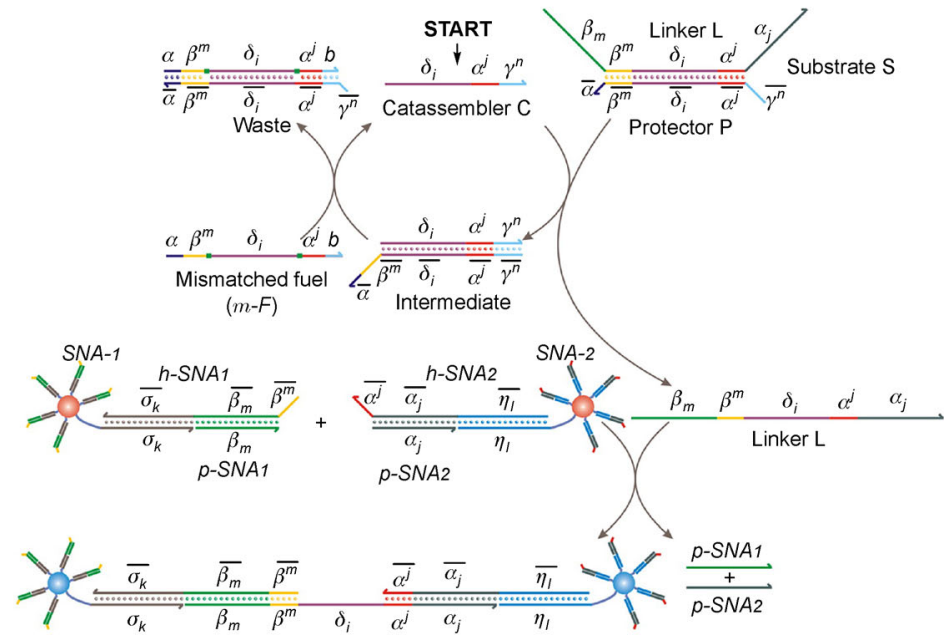

Figure 10. DNA-AuNP assembly applied to graphical representation SNP discrimination. (A) Graphical representation of SNP discrimination using DNA-fueled molecular machine-based DNA-AuNP assembly; reprinted with permission from Ref. [15], Copyright 2014 WILEY-VCH Verlag GmbH \& Co. KGaA, Weinheim. (B) Graphical representation of the integrating catalytic circuit with self-assembly of DNA-AuNP conjugates; reprinted with permission from Ref. [14], Copyright 2015 American Chemical Society. 
in any position of the target DNA, the integrated system exhibited obvious discrimination between the correct DNA and mutated DNA.

\section{Strand displacement introduced in gene regulation}

Toehold-mediated strand displacement reaction-based the regulation of gene expression

In the field's earliest stages, "synthetic biology" was expressed in the gene recombination technique by Hobom $\mathrm{B}$ in 1980 [85]. With the development of molecular systems biology, E. Kool mentioned the title "synthetic biology" again at the annual meeting of the American Chemical Society in 2000. E. Kool introduced the title for the synthesis of unnatural organic molecules in living systems. Three years later, "synthetic biology" was internationally defined as genetic engineering and engineering methods of artificial biological systems research based on system biology. A wide range of synthetic artificial biological gene circuits were designed and constructed by engineering principles and methods, such as toggle switches [86] and ring oscillators [87], which caused the rapid development of molecular counters [88], logic gates [89-92], sensor, and others.

A new regulation mechanism called riboregulator or toehold switches in synthetic biology [10] successfully combined the DNA/RNA strand displacement reaction and gene expression system. This mechanism has the potential to expand biosensing to gene regulation and disease gene diagnosis. Until this point, engineered riboregulators consist of a pair of homologous RNA: a transducer strand with an isolation of the ribosomal binding site (RBS), and a trans-acting RNA (taRNA) that

A Conventional riboregulator

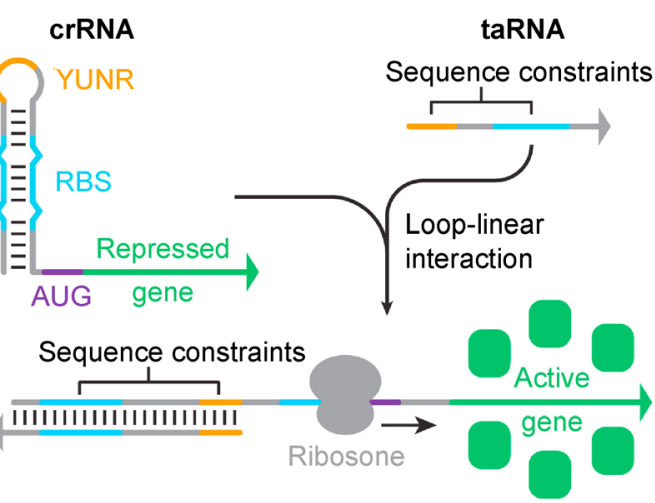

functions as a key of the switch that binds to the transducer strand to regulate gene expression of downstream mRNA and biological activity (Figure 11A) [10]. In the presence of taRNA of high binding energy, the YUNR domain first binds with taRNA as a toehold site and then exposes the RBS domain. Gene expression ensues by completing the RNA strand displacement reaction. Despite its strong activity, riboregulators are limited in diagnosis and treatment applications because of the sequence constraints of taRNA, which cannot distinguish real mRNA.

Green et al. [10] solved the problem in subsequent research by locking the start codon (AUG) sequence and placing the RBS in a loop region of a hairpin shaped RNA, which can prevent the identification of ribosome and the expression of signals. The corresponding trigger RNA eliminated the sequence constraints because of the $3 \mathrm{nt}$ bulge on the hairpin stem (Figure 11B). Ribosomes bind with RBS and activate translation only when the trigger RNA binds the toehold of switch RNA, which releases the domain of RBS and AUG and then restores mRNA to a linear state.

These systems realized the detection of functional mRNA in living cells, however, due to the uncertainty of the cell internal environment, extensive attention has been given to external detection for synthetic biology.

In vitro synthetic biology has been in existence for more than a decade. This field has made important contributions for understanding the fundamental biochemistry and application of a more complex genetic response network [93-96]. Keith Pardee et al. [97] applied the gene network related to the toehold-mediated strand displacement reaction of mRNA to the cell-free system and proposed a new concept that adopts synthetic

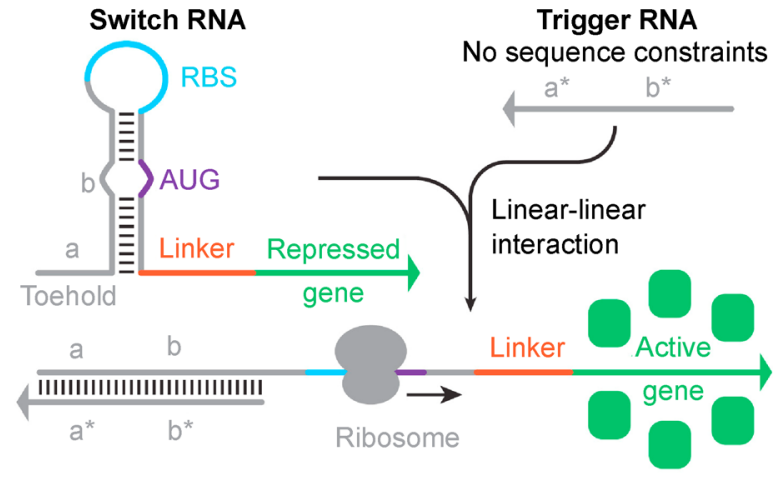

Figure 11. Schematics of RNA-based riboregulator biosensors and synthetic biology applications by RNA toeholdmediated strand displacement reaction. (A) Conventional riboregulators repress the gene by trapping the RBS through a hairpin structure, which is released under the existence of the trans-acting RNA (taRNA). (B) Toehold switches repress translation by locking the start codon (AUG) sequence and placing the RBS through the stem structure. In RNA-RNA interactions, the toehold reaction activates translation and aligns the hairpin structure via binding with a complementary RNA (trigger RNA); reprinted with permission from Ref. [10], Copyright 2014 Elsevier Inc. 
gene networks on paper (Figure 12). In this concept, by freeze-drying onto paper or other porous substrates, cellfree systems enable the created gene networks to be stable for long-term storage at room temperature. More importantly, the gene circuits are activated by simply adding water with the diagnosis of samples, such as target mRNA that can trigger RNA, synthetic RNA, or small molecules.

The paper-based sensors have achieved qualitative breakthrough applications in disease diagnosis, such as the Zika virus outbreak in the Americas. Pardee et al. [98] (Figure 13) created a platform of diagnostic sensors to rapidly and conveniently implement the visual low-cost detection of the Zika virus. The gene networks combined with the popular CRISPR-cas9 technology were used to increase diagnostic capabilities. This sensor based on the strand displacement reaction play an outstanding role in diagnostic applications, which can identify disease by determine the pathogen in a rapid and inexpensive way.

Conditional RNA interference (RNAi) applied to gene regulation

RNAi has emerged as a powerful technique for posttranscriptional gene regulation, which can use small RNAs knockdown specific gene expression simply and effectively without involving regulatory factors or specific proteins in diverse eukaryotes [99]. These small RNAs mainly include small interfering RNAs (siRNAs) and miRNAs, of which siRNAs are dominating regulatory molecules that function as guides for sequencespecific cleavage of mRNA by the RNA-induced silencing complex. Compared with chemically synthesized siRNAs, long double-stranded RNAs (dsRNAs) or short hairpin RNAs (shRNA) [100], which can be transcribed and cleaved by the RNase III enzyme Dicer to form functional siRNAs in vivo, can avoid unwanted cellular toxicity and obtain effective silencing. Therefore, they have great potential in the spatial and/or temporal regulation of RNAi for basic gene therapy application.

Unlike conventional RNAi in which dsRNAs are cleaved directly by Dicer enzyme, many methods are designed to engineer an RNA-based platform, which called a shRNA switch to control RNA interference. Such as Beisel CL, et al. designed a model-guided of ligandregulated RNAi by modular coupling of an aptamer, competing strand, and small hairpin RNA stem into a single component that programmable control gene expression [101]. Likewise, Sando et al. proposed a conditional RNAi pathway that indirectly regulates the desired siRNA formation and combines the concept of toehold-mediated strand displacement. This strategy comprises three parts: a synthetic hairpin-shaped RNA fused with the sense strand of siRNA (Hp-SS), the antisense strand of siRNA, and a single strand named trigger strand complementary to the loop region of Hp-SS (Figure 14A) [102]. By adjusting the stem region length of Hp-SS, which requires AS not to hybridize with Hp-SS directly without a trigger RNA, they used a 2'-O-methyl (2'-OMe)-modified regulatory stem region with 19 -mer length, which is long enough to obstruct the strand displacement reaction of Hp-SS and AS and can prevent Hp-SS causing RNAi. In this condition, only when the trigger strand was present, HP-SS could be opened and RNAi could be processed.

Later, the group of Yokobayashi developed a modified oligonucleotide-inducible RNAi (MONi-RNAi) [103] based on previous small-molecule inducible RNAi designs [104]. The designed RNA transcript called

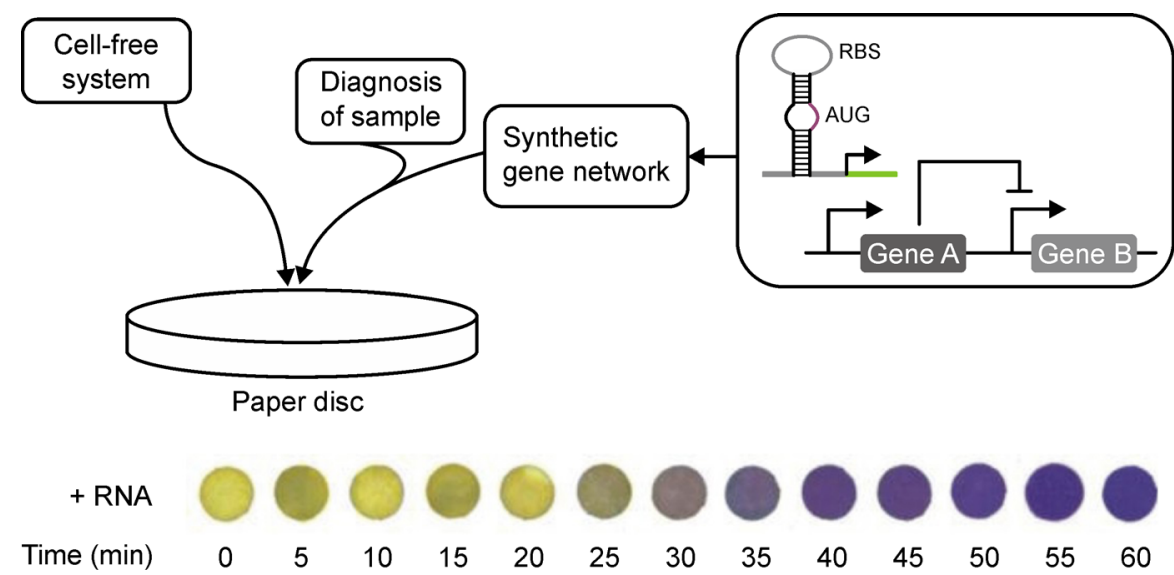

Figure 12. The creation of synthetic biology platforms on paper. The cell-free transcription and translation systems are combined with the created synthetic gene networks and then freeze-dried onto paper discs to establish stability for long-term extracellular storage of synthetic gene network. The synthetic biology platforms can be observed for the corresponding response when response factor RNA or small molecules are added. 

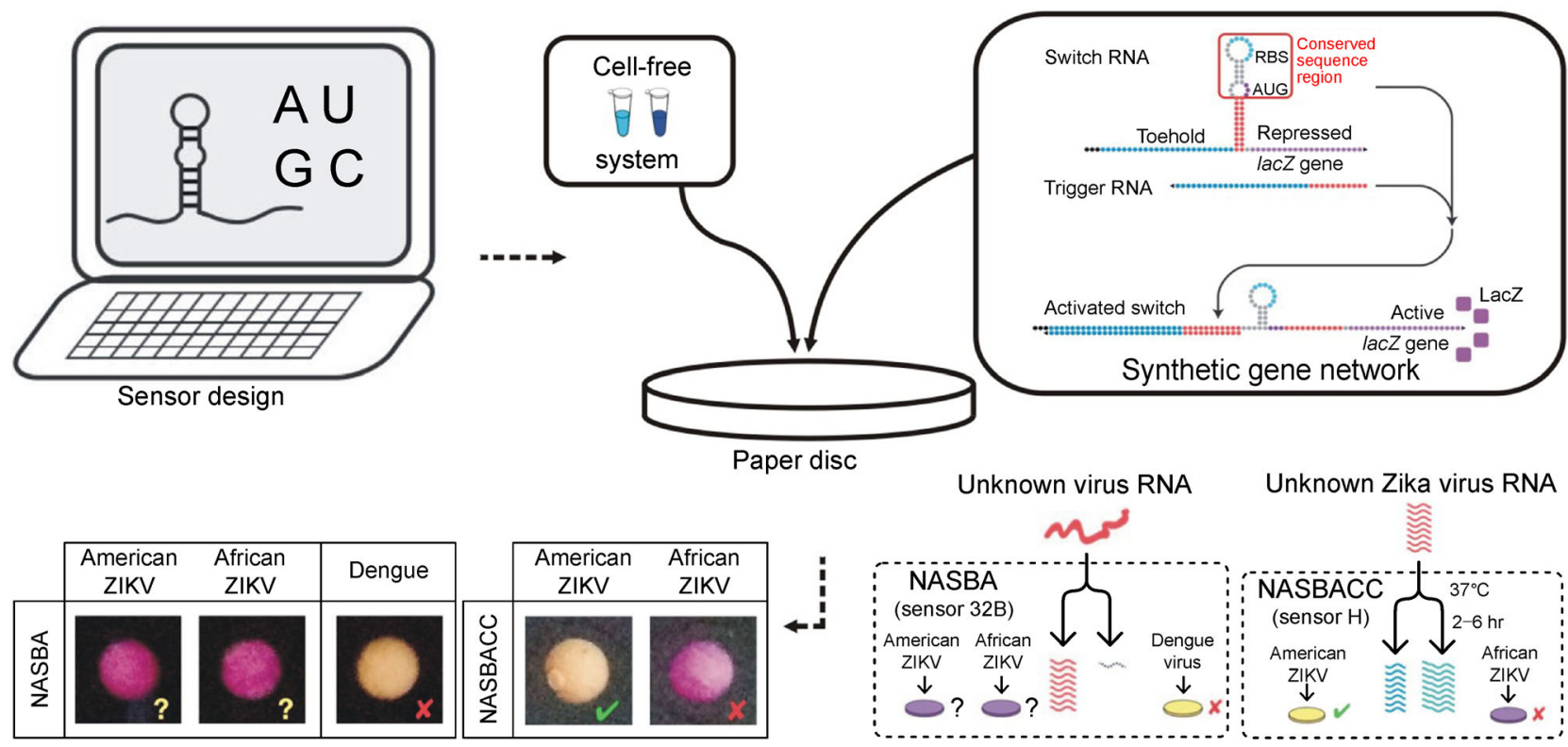

Figure 13. The workflow of paper-based RNA toehold displacement sensors for Zika virus detection. The sequence of toehold switches of paper-based RNA sensor were designed to the desired device parameters in silico, combined with the RNA sensor and cell-free protein expression system embedded into paper discs, and freeze-dried for stable diagnostic. The Zika virus RNA can be selected by a color change in the paper, which were isothermally amplified to a proper concentration via NASBA and NASBACC, and used to rehydrate the paper sensor for activating reaction; reprinted with permission from Ref. [98], Copyright 2016 Elsevier Inc.

A

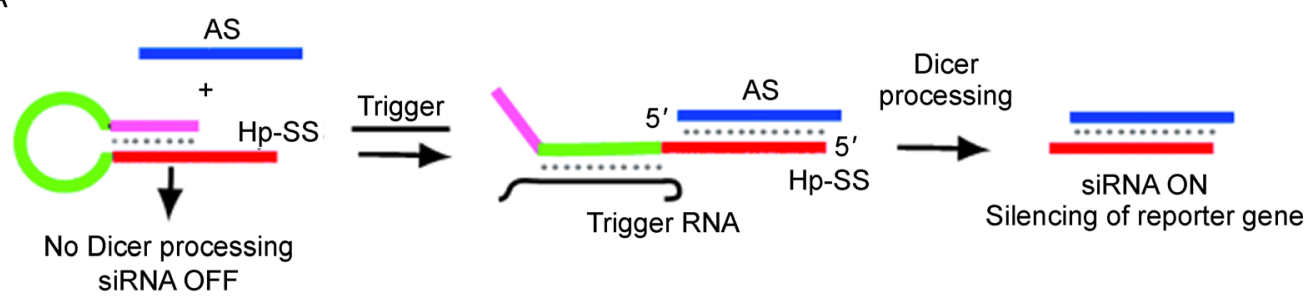

B

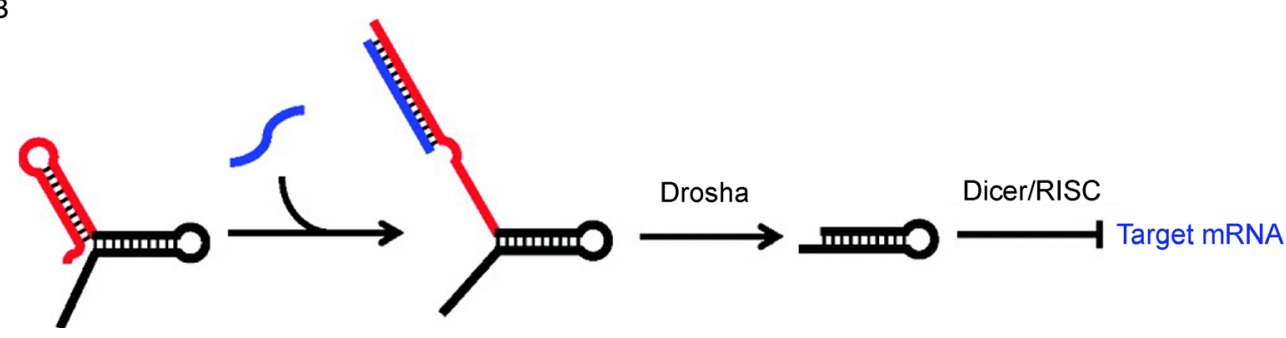

Figure 14. Conditional RNAi regulated by strand displacement. (A) Schematic illustrations of the designed activatable siRNA system; reprinted with permission from Ref. [101]. Copyright 2009 Wiley-VCH Verlag GmbH \& Co. KGaA, Weinheim. (B) Schematic illustration of MONi-RNAi strategy and mechanism; reprinted with permission from Ref. [102], Copyright 2011 American Chemical Society.

moniRNA contained two stem-loop domains, one of which was a RNAi effector processed to repress EGFP expression, and another part of the moniRNA was a MON sensor that could react with $2^{\prime}$-OMe-modified inducer
RNA via toehold-mediated strand displacement processing and then release the 5'-long single strand, which is the necessary condition for Dorsha cleavage to process RNAi (Figure 14B) [103]. They screened many 

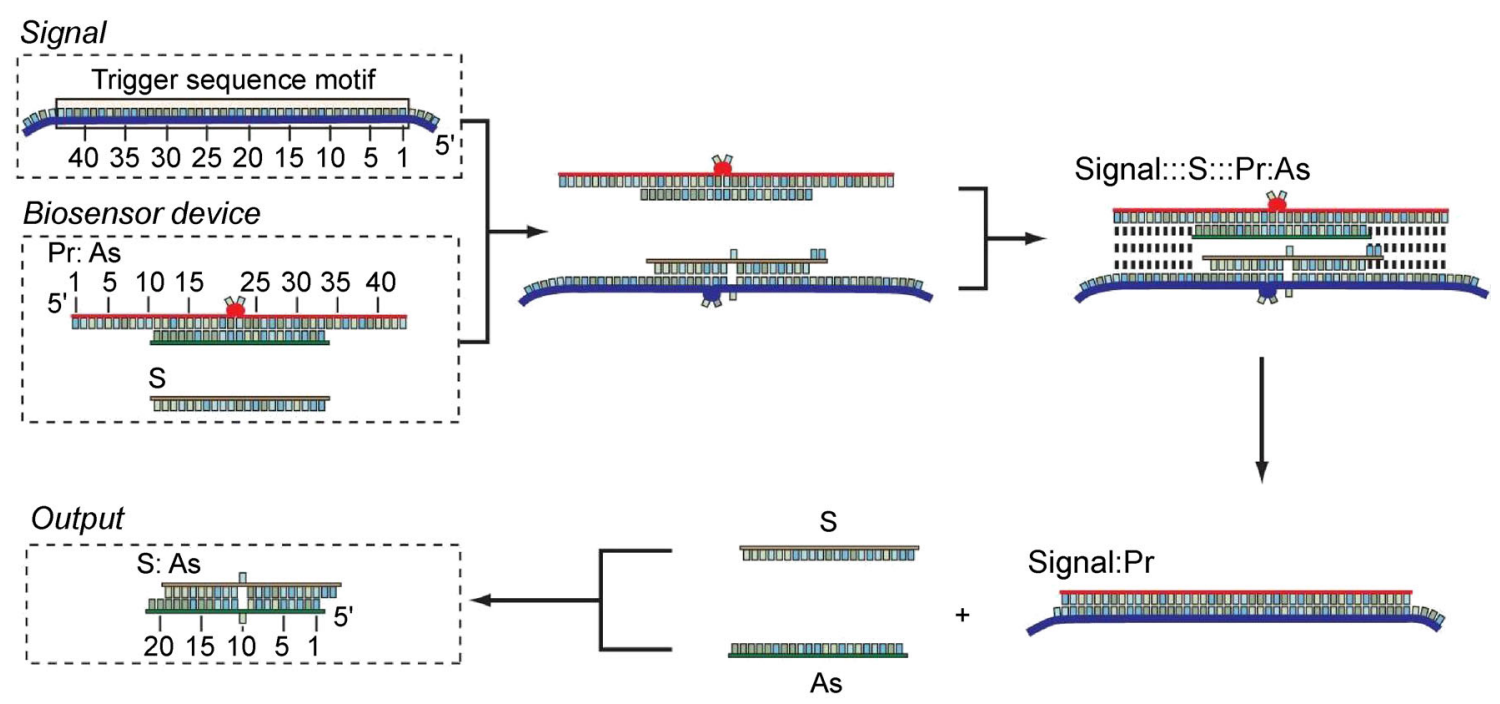

Figure 15. The biosensor device for mRNA signal and the signal transduction process. The mRNA signal contains a "trigger-sequence motif" 43-nt long. The biosensor device consists of a Pr:As duplex which "protecting" (Pr) strand is pre-annealed to an "antisense" (As) strand with two 10-nt single-stranded overhangs, and a single "sense" (S) strand. The Pr:As duplex can interact with the trigger-sequence motif. After the strand migration, the released As strand hybridizes with the single-stranded $S$ strand to form a canonical siRNA duplex S: As; reprinted with permission from Ref. [104], Copyright 2010 Oxford University Press.

sequences of MON sensor domain with different lengths and free energies and then integrated two orthogonal MONi-RNAi systems to silence two genes respectively in HEK293 cells.

Furthermore, Xie and co-workers constructed and implemented a biosensor that "transduce" mRNA levels into bioactive, siRNA molecules via RNA strand displacement in a cell-free Drosophila embryo lysate, which allows a level of control that is impossible to achieve in live cells, and yet retains many properties of the cytoplasm (Figure 15) [105]. The nucleic acid biosensor consists of a partially double-stranded RNA duplex (Pr: As) and a single-stranded RNA duplex (S). The Pr strand is complementary to the trigger-sequence motif, while the As strand is complementary to the central half of the $\mathrm{Pr}$ strand, leaving two 10-nt single-stranded toehold overhangs. Finally the single-stranded As molecule will free to hybridize to the complementary $\mathrm{S}$ strand, forming a functional siRNA (S:As), which is used to regulate the subsequent expression of target gene.

These works indicated the possibility of using toeholdmediated strand displacement to establish RNAi-based gene logic circuits or biosensor, as well as regulate gene expression in a native biological setting for various applications such as gene therapy.

\section{CONCLUSIONS}

The toehold-mediated strand displacement has proved its crucial role in dynamic DNA nanotechnology. As its key components, predictable Watson-Crick base pairing and tunable toehold enable it to be well understood for theoretical modeling and experimental control. In this case, toehold-mediated strand displacement is endowed with outstanding modularity and scalability and has been successfully used as building blocks to construct exquisite molecular devices, assembly structures, complex functional circuits and reaction networks. In addition, toeholdmediated strand displacement also shows great compatibility and potential in biotechnology since it has been employed for biomedical applications such as SNP discrimination and gene-induced disease detection, even for gene expression regulation. In the near future, toeholdmediated strand displacement will build a good friendship with mankind life.

\section{ACKNOWLEDGEMENTS}

We would like to thank the National Natural Science Foundation of China (Nos. 91427304, 21434007, 21574122, 51573175, and 21404098), the National Basic Research Program of China (No. 2012CB821500), and the Fundamental Research Funds for the Central Universities (WK3450000002 and WK2060200017) for their financial support.

\section{COMPLIANCE WITH ETHICS GUIDELINES}

The authors Yijun Guo, Bing Wei, Shiyan Xiao, Dongbao Yao, Hui Li, Huaguo $\mathrm{Xu}$, Tingjie Song, Xiang Li and Haojun Liang declare no competing financial and personal relationships with other people or organizations that can inappropriately influence our work. There is no professional or other personal interest of any nature or kind in any product, 
service and/or company that could be construed as influencing the position presented in, or the review of, the manuscript titled "Recent Advances in Molecular Machines Based on Toehold-mediated Strand Displacement Reaction".

This article does not contain any studies with human or animal subjects performed by any of the authors.

\section{REFERENCES}

1. Yurke, B., Turberfield, A. J., Mills, A. P. Jr, Simmel, F. C. and Neumann, J. L. (2000) A DNA-fuelled molecular machine made of DNA. Nature, 406, 605-608

2. Zhang, D. Y. and Seelig, G. (2011) Dynamic DNA nanotechnology using strand-displacement reactions. Nat. Chem., 3, 103-113

3. Pan, J., Li, F., Cha, T. G., Chen, H. and Choi, J. H. (2015) Recent progress on DNA based walkers. Curr. Opin. Biotechnol., 34, 56-64

4. You, M., Peng, L., Shao, N., Zhang, L., Qiu, L., Cui, C. and Tan, W. (2014) DNA "nano-claw": logic-based autonomous cancer targeting and therapy. J. Am. Chem. Soc., 136, 1256-1259

5. Rudchenko, M., Taylor, S., Pallavi, P., Dechkovskaia, A., Khan, S., Butler, V. P. Jr, Rudchenko, S. and Stojanovic, M. N. (2013) Autonomous molecular cascades for evaluation of cell surfaces. Nat. Nanotechnol., 8, 580-586

6. Andersen, E. S., Dong, M., Nielsen, M. M., Jahn, K., Subramani, R., Mamdouh, W., Golas, M. M., Sander, B., Stark, H., Oliveira, C. L. P., et al. (2009) Self-assembly of a nanoscale DNA box with a controllable lid. Nature, 459, 73-76

7. Parolini, L., Kotar, J., Di Michele, L. and Mognetti, B. M. (2016) Controlling self-assembly kinetics of DNA-functionalized liposomes using toehold exchange mechanism. ACS Nano, 10, 2392-2398

8. Hellyer, T. J. and Nadeau, J. G. (2004) Strand displacement amplification: a versatile tool for molecular diagnostics. Expert Rev. Mol. Diagn., 4, 251-261

9. Kushwaha, M., Rostain, W., Prakash, S., Duncan, J. N. and Jaramillo, A. (2016) Using RNA as molecular code for programming cellular function. ACS Synth. Biol., 5, 795-809

10. Green, A. A., Silver, P. A., Collins, J. J. and Yin, P. (2014) Toehold switches: de-novo-designed regulators of gene expression. Cell, 159, 925-939

11. Qian, L. and Winfree, E. (2011) Scaling up digital circuit computation with DNA strand displacement cascades. Science, 332, 1196-1201

12. Song, T. and Liang, H. (2012) Synchronized assembly of gold nanoparticles driven by a dynamic DNA-fueled molecular machine. J. Am. Chem. Soc., 134, 10803-10806

13. Xu, H., Deng, W., Huang, F., Xiao, S., Liu, G. and Liang, H. (2014) Enhanced DNA toehold exchange reaction on a chip surface to discriminate single-base changes. Chem. Commun. (Camb.), 50, 14171-14174

14. Yao, D., Song, T., Sun, X., Xiao, S., Huang, F. and Liang, H. (2015) Integrating DNA-strand-displacement circuitry with self-assembly of spherical nucleic acids. J. Am. Chem. Soc., 137, 14107-14113

15. Song, T., Xiao, S., Yao, D., Huang, F., Hu, M. and Liang, H. (2014) An efficient DNA-fueled molecular machine for the discrimination of single-base changes. Adv. Mater., 26, 6181-6185

16. Zhang, D. Y., Turberfield, A. J., Yurke, B. and Winfree, E. (2007) Engineering entropy-driven reactions and networks catalyzed by DNA. Science, 318, 1121-1125
17. Zhang, D. Y. and Winfree, E. (2009) Control of DNA strand displacement kinetics using toehold exchange. J. Am. Chem. Soc., 131, 17303-17314

18. Srinivas, N., Ouldridge, T. E., Šulc, P., Schaeffer, J. M., Yurke, B., Louis, A. A., Doye, J. P. K. and Winfree, E. (2013) On the biophysics and kinetics of toehold-mediated DNA strand displacement. Nucleic Acids Res., 41, 10641-10658

19. Machinek, R. R. F., Ouldridge, T. E., Haley, N. E. C., Bath, J. and Turberfield, A. J. (2014) Programmable energy landscapes for kinetic control of DNA strand displacement. Nat. Commun., 5, 5324

20. Nakayama, S., Yan, L. and Sintim, H. O. (2008) Junction probessequence specific detection of nucleic acids via template enhanced hybridization processes. J. Am. Chem. Soc., 130, 12560-12561

21. Xing, Y., Yang, Z. and Liu, D. (2011) A responsive hidden toehold to enable controllable DNA strand displacement reactions. Angew. Chem. Int. Ed. Engl., 50, 11934-11936

22. Huang, F., You, M., Han, D., Xiong, X., Liang, H. and Tan, W. (2013) DNA branch migration reactions through photocontrollable toehold formation. J. Am. Chem. Soc., 135, 7967-7973

23. Hemphill, J. and Deiters, A. (2013) DNA computation in mammalian cells: microRNA logic operations. J. Am. Chem. Soc., 135, 1051210518

24. Prokup, A., Hemphill, J., Liu, Q. and Deiters, A. (2015) Optically controlled signal amplification for DNA computation. ACS Synth. Biol., 4, 1064-1069

25. Amodio, A., Zhao, B., Porchetta, A., Idili, A., Castronovo, M., Fan, C. and Ricci, F. (2014) Rational design of pH-controlled DNA strand displacement. J. Am. Chem. Soc., 136, 16469-16472

26. Chen, X. (2012) Expanding the rule set of DNA circuitry with associative toehold activation. J. Am. Chem. Soc., 134, 263-271

27. Zhu, J., Zhang, L., Zhou, Z., Dong, S. and Wang, E. (2014) Aptamerbased sensing platform using three-way DNA junction-driven strand displacement and its application in DNA logic circuit. Anal. Chem., $86,312-316$

28. Zhu, J., Zhang, L., Dong, S. and Wang, E. (2013) Four-way junctiondriven DNA strand displacement and its application in building majority logic circuit. ACS Nano, 7, 10211-10217

29. Genot, A. J., Zhang, D. Y., Bath, J. and Turberfield, A. J. (2011) Remote toehold: a mechanism for flexible control of DNA hybridization kinetics. J. Am. Chem. Soc., 133, 2177-2182

30. Bommarito, S., Peyret, N. and SantaLucia, J. Jr (2000) Thermodynamic parameters for DNA sequences with dangling ends. Nucleic Acids Res., 28, 1929-1934

31. SantaLucia, J. Jr and Hicks, D. (2004) The thermodynamics of DNA structural motifs. Annu. Rev. Biophys. Biomol. Struct., 33, 415440

32. Wang, C., Bae, J. H. and Zhang, D. Y. (2016) Native characterization of nucleic acid motif thermodynamics via non-covalent catalysis. Nat. Commun., 7, 10319

33. Protozanova, E., Yakovchuk, P. and Frank-Kamenetskii, M. D. (2004) Stacked-unstacked equilibrium at the nick site of DNA. J. Mol. Biol., 342, 775-785

34. Pyshnyi, D. V. and Ivanova, E. M. (2002) Thermodynamic parameters of coaxial stacking on stacking hybridization of oligodeoxyribonucleotides. Russ. Chem. Bull., 51, 1145-1155

35. Yurke, B. and Mills, A. P. (2003) Using DNA to power nanostructures. Genet. Program. Evolvable Mach., 4, 111-122

36. Yang, X., Tang, Y., Traynor, S. M. and Li, F. (2016) Regulation of 
DNA strand displacement using allosteric DNA toehold. J. Am. Chem. Soc., 138, 14076-14082

37. Khimji, I., Shin, J. and Liu, J. (2013) DNA duplex stabilization in crowded polyanion solutions. Chem. Commun. (Camb.), 49, 13061308

38. Smith, B. D. and Liu, J. (2010) Assembly of DNA-functionalized nanoparticles in alcoholic solvents reveals opposite thermodynamic and kinetic trends for DNA hybridization. J. Am. Chem. Soc., 132, $6300-6301$

39. Zhang, T., Shang, C., Duan, R., Hakeem, A., Zhang, Z., Lou, X. and Xia, F. (2015) Polar organic solvents accelerate the rate of DNA strand replacement reaction. Analyst, 140, 2023-2028

40. Dave, N. and Liu, J. (2010) Fast molecular beacon hybridization in organic solvents with improved target specificity. J. Phys. Chem. B, 114, 15694-15699

41. Kang, D., Duan, R., Tan, Y., Hong, F., Wang, B., Chen, Z., Xu, S., Lou, X., Wei, W., Yurke, B. and Xia, F. (2014) Speeding up the selfassembly of a DNA nanodevice using a variety of polar solvents. Nanoscale, 6, 14153-14157

42. Wu, L. R., Wang, J. S., Fang, J. Z., Evans, E. R., Pinto, A., Pekker, I., Boykin, R., Ngouenet, C., Webster, P. J., Beechem, J., et al. (2015) Continuously tunable nucleic acid hybridization probes. Nat. Methods, 12, 1191-1196

43. Mirkin, C. A., Letsinger, R. L., Mucic, R. C. and Storhoff, J. J. (1996) A DNA-based method for rationally assembling nanoparticles into macroscopic materials. Nature, 382, 607-609

44. Alivisatos, A. P., Johnsson, K. P., Peng, X., Wilson, T. E., Loweth, C. J., Bruchez, M. P. Jr and Schultz, P. G. (1996) Organization of "nanocrystal molecules" using DNA. Nature, 382, 609-611

45. Nykypanchuk, D., Maye, M. M., van der Lelie, D. and Gang, O. (2008) DNA-guided crystallization of colloidal nanoparticles. Nature, 451, 549-552

46. Park, S. Y., Lytton-Jean, A. K. R., Lee, B., Weigand, S., Schatz, G. C. and Mirkin, C. A. (2008) DNA-programmable nanoparticle crystallization. Nature, 451, 553-556

47. Macfarlane, R. J., Jones, M. R., Senesi, A. J., Young, K. L., Lee, B., Wu, J. and Mirkin, C. A. (2010) Establishing the design rules for DNA-mediated programmable colloidal crystallization. Angew. Chem. Int. Ed. Engl., 49, 4589-4592

48. O'Brien, M. N., Jones, M. R., Lee, B. and Mirkin, C. A. (2015) Anisotropic nanoparticle complementarity in DNA-mediated cocrystallization. Nat. Mater., 14, 833-839

49. Jones, M. R., Seeman, N. C. and Mirkin, C. A. (2015) Programmable materials and the nature of the DNA bond. Science, 347, 1260901

50. Sharma, J., Chhabra, R., Cheng, A., Brownell, J., Liu, Y. and Yan, H. (2009) Control of self-assembly of DNA tubules through integration of gold nanoparticles. Science, 323, 112-116

51. Shen, X., Song, C., Wang, J., Shi, D., Wang, Z., Liu, N. and Ding, B. (2012) Rolling up gold nanoparticle-dressed DNA origami into threedimensional plasmonic chiral nanostructures. J. Am. Chem. Soc., 134, $146-149$

52. Lan, X., Lu, X., Shen, C., Ke, Y., Ni, W. and Wang, Q. (2015) Au nanorod helical superstructures with designed chirality. J. Am. Chem. Soc., $137,457-462$

53. Tian, Y., Wang, T., Liu, W., Xin, H. L., Li, H., Ke, Y., Shih, W. M. and Gang, O. (2015) Prescribed nanoparticle cluster architectures and lowdimensional arrays built using octahedral DNA origami frames. Nat. Nanotechnol., 10, 637-644
54. Liu, W., Halverson, J., Tian, Y., Tkachenko, A. V. and Gang, O. (2016) Self-organized architectures from assorted DNA-framed nanoparticles. Nat. Chem., 8, 867-873

55. Rogers, W. B., Shih, W. M. and Manoharan, V. N. (2016) Using DNA to program the self-assembly of colloidal nanoparticles and microparticles. Nat Rev Mater., 1, 16008

56. Saha, K., Agasti, S. S., Kim, C., Li, X. and Rotello, V. M. (2012) Gold nanoparticles in chemical and biological sensing. Chem. Rev., 112, 2739-2779

57. Wilner, O. I. and Willner, I. (2012) Functionalized DNA nanostructures. Chem. Rev., 112, 2528-2556

58. Zhao, Y., Chen, F., Li, Q., Wang, L. and Fan, C. (2015) Isothermal amplification of nucleic acids. Chem. Rev., 115, 12491-12545.

59. Rosi, N. L. and Mirkin, C. A. (2005) Nanostructures in biodiagnostics. Chem. Rev., 105, 1547-1562

60. Liu, J., Cao, Z. and Lu, Y. (2009) Functional nucleic acid sensors. Chem. Rev., 109, 1948-1998

61. Wu, P., Hwang, K., Lan, T. and Lu, Y. (2013) A DNAzyme-gold nanoparticle probe for uranyl ion in living cells. J. Am. Chem. Soc., $135,5254-5257$

62. Liang, H., Zhang, X.-B., Lv, Y., Gong, L., Wang, R., Zhu, X., Yang, R. and Tan, W. (2014) Functional DNA-containing nanomaterials: cellular applications in biosensing, imaging, and targeted therapy. Acc. Chem. Res., 47, 1891-1901

63. Saiki, R. K., Gelfand, D. H., Stoffel, S., Scharf, S. J., Higuchi, R., Horn, G. T., Mullis, K. B. and Erlich, H. A. (1988) Primer-directed enzymatic amplification of DNA with a thermostable DNA polymerase. Science, 239, 487-491

64. Gunderson, K. L., Steemers, F. J., Lee, G., Mendoza, L. G. and Chee, M. S. (2005) A genome-wide scalable SNP genotyping assay using microarray technology. Nat. Genet., 37, 549-554

65. Schena, M., Shalon, D., Davis, R. W. and Brown, P. O. (1995) Quantitative monitoring of gene expression patterns with a complementary DNA microarray. Science, 270, 467-470

66. Mardis, E. R. (2008) Next-generation DNA sequencing methods. Annu. Rev. Genomics Hum. Genet., 9, 387-402

67. Zhang, D. Y., Chen, S. X. and Yin, P. (2012) Optimizing the specificity of nucleic acid hybridization. Nat. Chem., 4, 208-214

68. Chen, S. X., Zhang, D. Y. and Seelig, G. (2013) Conditionally fluorescent molecular probes for detecting single base changes in double-stranded DNA. Nat. Chem., 5, 782-789

69. Wang, J. S. and Zhang, D. Y. (2015) Simulation-guided DNA probe design for consistently ultraspecific hybridization. Nat. Chem., 7, 545-553

70. Chen, S. X. and Seelig, G. (2016) An engineered kinetic amplification mechanism for single nucleotide variant discrimination by DNA hybridization probes. J. Am. Chem. Soc., 138, 5076-5086

71. Karim, K., Taylor, J. D., Cullen, D. C., Swann, M. J. and Freeman, N. J. (2007) Measurement of conformational changes in the structure of transglutaminase on binding calcium ions using optical evanescent dual polarisation interferometry. Anal. Chem., 79, 3023-3031

72. Mashaghi, A., Swann, M., Popplewell, J., Textor, M. and Reimhult, E. (2008) Optical anisotropy of supported lipid structures probed by waveguide spectroscopy and its application to study of supported lipid bilayer formation kinetics. Anal. Chem., 80, 3666-3676

73. Xu, P., Huang, F. and Liang, H. (2013) Real-time study of a DNA strand displacement reaction using dual polarization interferometry. Biosens. Bioelectron., 41, 505-510 
74. Tang, W., Wang, D., Xu, Y., Li, N. and Liu, F. (2012) A self-assembled DNA nanostructure-amplified quartz crystal microbalance with dissipation biosensing platform for nucleic acids. Chem. Commun. (Camb.), 48, 6678-6680

75. Zhao, Y., Wang, H., Tang, W., Hu, S., Li, N. and Liu, F. (2015) An in situ assembly of a DNA-streptavidin dendrimer nanostructure: a new amplified quartz crystal microbalance platform for nucleic acid sensing. Chem. Commun. (Camb.), 51, 10660-10663

76. Tang, W., Hu, S., Wang, H., Zhao, Y., Li, N. and Liu, F. (2014) A universal molecular translator for non-nucleic acid targets that enables dynamic DNA assemblies and logic operations. Chem. Commun. (Camb.), 50, 14352-14355

77. Wang, D., Chen, G., Wang, H., Tang, W., Pan, W., Li, N. and Liu, F. (2013) A reusable quartz crystal microbalance biosensor for highly specific detection of single-base DNA mutation. Biosens. Bioelectron., 48, 276-280

78. Li, H., Xiao, S., Yao, D., Lam, M. H.-W. and Liang, H. (2015) A smart DNA-gold nanoparticle probe for detecting single-base changes on the platform of a quartz crystal microbalance. Chem. Commun. (Camb.), $51,4670-4673$

79. Tyagi, S. and Kramer, F. R. (1996) Molecular beacons: probes that fluoresce upon hybridization. Nat. Biotechnol., 14, 303-308

80. Papadopoulou, E. and Bell, S. E. J. (2011) Label-free detection of single-base mismatches in DNA by surface-enhanced Raman spectroscopy. Angew. Chem. Int. Ed. Engl., 50, 9058-9061

81. Elghanian, R., Storhoff, J. J., Mucic, R. C., Letsinger, R. L. and Mirkin, C. A. (1997) Selective colorimetric detection of polynucleotides based on the distance-dependent optical properties of gold nanoparticles. Science, 277, 1078-1081

82. Taton, T. A., Mirkin, C. A. and Letsinger, R. L. (2000) Scanometric DNA array detection with nanoparticle probes. Science, 289, 17571760

83. Yao, D., Song, T., Zheng, B., Xiao, S., Huang, F. and Liang, H. (2015) The combination of gold nanorods and nanoparticles with DNA nanodevices for logic gates construction. Nanotechnology, 26, 425601

84. Yao, D., Li, H., Guo, Y., Zhou, X., Xiao, S. and Liang, H. (2016) A $\mathrm{pH}$-responsive DNA nanomachine-controlled catalytic assembly of gold nanoparticles. Chem. Commun. (Camb.), 52, 7556-7559

85. Hobom, B. (1980) Surgery of genes - at the doorstep of synthetic biology. Med. Klin., 75, 14-21

86. Gardner, T. S., Cantor, C. R. and Collins, J. J. (2000) Construction of a genetic toggle switch in Escherichia coli. Nature, 403, 339-342

87. Elowitz, M. B. and Leibler, S. (2000) A synthetic oscillatory network of transcriptional regulators. Nature, 403, 335-338

88. Friedland, A. E., Lu, T. K., Wang, X., Shi, D., Church, G. and Collins, J. J. (2009) Synthetic gene networks that count. Science, 324, 11991202

89. Ausländer, S., Ausländer, D., Müller, M., Wieland, M. and Fussenegger, M. (2012) Programmable single-cell mammalian biocomputers. Nature, 487, 123-127

90. Moon, T. S., Lou, C., Tamsir, A., Stanton, B. C. and Voigt, C. A.
(2012) Genetic programs constructed from layered logic gates in single cells. Nature, 491, 249-253

91. Siuti, P., Yazbek, J. and Lu, T. K. (2013) Synthetic circuits integrating logic and memory in living cells. Nat. Biotechnol., 31, 448-452

92. Win, M. N. and Smolke, C. D. (2008) Higher-order cellular information processing with synthetic RNA devices. Science, 322, $456-460$

93. Hong, S. H., Ntai, I., Haimovich, A. D., Kelleher, N. L., Isaacs, F. J. and Jewett, M. C. (2014) Cell-free protein synthesis from a release factor 1 deficient Escherichia coli activates efficient and multiple sitespecific nonstandard amino acid incorporation. ACS Synth. Biol., 3, 398-409

94. Karzbrun, E., Tayar, A. M., Noireaux, V. and Bar-Ziv, R. H. (2014) Programmable on-chip DNA compartments as artificial cells. Science, $345,829-832$

95. Sun, Z. Z., Yeung, E., Hayes, C. A., Noireaux, V. and Murray, R. M. (2014) Linear DNA for rapid prototyping of synthetic biological circuits in an Escherichia coli based TX-TL cell-free system. ACS Synth. Biol., 3, 387-397

96. Takahashi, M. K., Chappell, J., Hayes, C. A., Sun, Z. Z., Kim, J., Singhal, V., Spring, K. J., Al-Khabouri, S., Fall, C. P., Noireaux, V., et al. (2015) Rapidly characterizing the fast dynamics of RNA genetic circuitry with cell-free transcription-translation (TX-TL) systems. ACS Synth. Biol., 4, 503-515

97. Pardee, K., Green, A. A., Ferrante, T., Cameron, D. E., DaleyKeyser, A., Yin, P. and Collins, J. J. (2014) Paper-based synthetic gene networks. Cell, 159, 940-954

98. Pardee, K., Green, A. A., Takahashi, M. K., Braff, D., Lambert, G., Lee, J. W., Ferrante, T., Ma, D., Donghia, N., Fan, M., et al. (2016) Rapid, low-cost detection of Zika virus using programmable biomolecular components. Cell, 165, 1255-1266

99. Hannon, G. J. and Rossi, J. J. (2004) Unlocking the potential of the human genome with RNA interference. Nature, 431, 371-378

100. Wiznerowicz, M., Szulc, J. and Trono, D. (2006) Tuning silence: conditional systems for RNA interference. Nat. Methods, 3, 682688

101. Beisel, C. L., Bayer, T. S., Hoff, K. G. and Smolke, C. D. (2008) Model-guided design of ligand-regulated RNAi for programmable control of gene expression. Mol. Syst. Biol., 4, 224

102. Masu, H., Narita, A., Tokunaga, T., Ohashi, M., Aoyama, Y. and Sando, S. (2009) An activatable siRNA probe: trigger-RNAdependent activation of RNAi function. Angew. Chem. Int. Ed. Engl., 48, 9481-9483

103. Kumar, D., Kim, S. H. and Yokobayashi, Y. (2011) Combinatorially inducible RNA interference triggered by chemically modified oligonucleotides. J. Am. Chem. Soc., 133, 2783-2788

104. Kumar, D., An, C.-I. and Yokobayashi, Y. (2009) Conditional RNA interference mediated by allosteric ribozyme. J. Am. Chem. Soc., 131, 13906-13907

105. Xie, Z., Liu, S. J., Bleris, L. and Benenson, Y. (2010) Logic integration of mRNA signals by an RNAi-based molecular computer. Nucleic Acids Res., 38,2692-2701 